\title{
Universal single-residue terminal labels for fluorescent live cell imaging of microproteins
}

Lorenzo Lafranchi, Dörte Schlesinger, Kyle Kimler, Simon J Elsässer*

Science for Life Laboratory, Department of Medical Biochemistry and Biophysics, Division of Genome Biology, Karolinska Institutet, Stockholm, Sweden

Ming Wai Lau Centre for Reparative Medicine, Stockholm node, Karolinska Institutet, Stockholm, Sweden

*Corresponding Author: simon.elsasser@scilifelab.se

\section{SUPPLEMENTARY INFORMATION}

MATERIALS AND METHODS

SUPPLEMENTARY FIGURES 1-13

SUPPLEMENTARY MOVIES 1-2

SUPPLEMENTARY REFERENCES

UNCROPPED SOURCE IMAGES 


\section{Materials and Methods}

\section{DNA constructs}

Plasmids were generated using the pAS plasmid backbone (E400) described previously. ${ }^{1}$ The Intein-FKBP cassette was ordered at Thermo Fisher, whereas sORFs sequences were synthesized by Twist Bioscience. All DNA constructs were verified by Sanger sequencing.

\begin{tabular}{|c|c|c|}
\hline ID & Name & $\begin{array}{l}\text { Reference/ } \\
\text { Repository }\end{array}$ \\
\hline E400 & pAS1_4x7SKPylT_EF1_In_IRES_Bsd & Addgene 140008 \\
\hline E402 & pAS1_4xh7SKPylT_EF1_GFP150TAG_IRES_Bsd & $\begin{array}{l}\text { Meineke, 2018; } \\
\text { Addgene } 140015\end{array}$ \\
\hline E407 & pAS1_4xh7SKPylT_EF1_PylRS AF_IRES_Puro & Addgene 140023 \\
\hline B106 & pAS1_EF1_GFP 150TAG_TGA_IRES_Bsd & $\begin{array}{l}\text { Meineke, 2018; } \\
\text { Addgene } 154765\end{array}$ \\
\hline L264.2 & pAS1_4xh7SKPylT_EF1_GFP & \\
\hline L268.1 & pAS1_4xh7SKPylT_EF1_GFP-150TAG & \\
\hline K038.2 & pAS1_4x7SKPylT_EF1_Ub-BsmBI & Addgene 162801 \\
\hline K039.2 & pAS1_4x7SKPylT_EF1_Ub-BsmBI-HA & Addgene 162802 \\
\hline K061.3 & pAS1_4x7SKPylT_EF1_Ub(G75,76A)-BsmBI & Addgene 162803 \\
\hline K062.2 & pAS1_4x7SKPylT_EF1_Ub-GFP & \\
\hline K063 & pAS1_4x7SKPylT_EF1_Ub(G75,76A)-GFP & \\
\hline K064.1 & pAS1_4x7SKPylT_EF1_Ub-GFP-150TAG & \\
\hline K065.1 & pAS1_4x7SKPylT_EF1_Ub-*GFP & \\
\hline K066.2 & pAS1_4x7SKPylT_EF1_Ub(G75,76A)-*GFP & \\
\hline L262.2 & pAS1_4xh7SKPylT_EF1_BsmBI_Intein_DD & Addgene 162810 \\
\hline L263.1 & pAS1_4xh7SKPylT_EF1_BsmBI_(C1A)-Intein_DD & Addgene 162811 \\
\hline L269.1 & pAS1_4xh7SKPylT_EF1_GFP_Intein_DD & \\
\hline
\end{tabular}




\begin{tabular}{|c|c|c|}
\hline L270.3 & pAS1_4xh7SKPylT_EF1_GFP_(C1A)-Intein_DD & \\
\hline L266.3 & pAS1_4xh7SKPylT_EF1_GFP*_Intein_DD & Addgene 162812 \\
\hline L267.2 & pAS1_4xh7SKPylT_EF1_GFP*_(C1A)-Intein_DD & \\
\hline L271.1 & pAS1_4xh7SKPylT_EF1_GFP-150TAG_Intein_DD & \\
\hline K037.2 & pAS1_4x7SKPylT_EF1_Ub-*NoBody-HA & \\
\hline K035.1 & pAS1_4x7SKPylT_EF1_Ub-*SMIM38-HA & \\
\hline K044.1 & pAS1_4x7SKPylT_EF1_*PIGBOS-HA & \\
\hline K075.2 & pAS1_4x7SKPylT_EF1_Ub(G75,76A)-*PIGBOS-HA & \\
\hline $\mathrm{L} 273.2$ & pAS1_4xh7SKPylT_EF1_NoBody*_Intein_DD & Addgene 162813 \\
\hline $\mathrm{L} 275.2$ & pAS1_4xh7SKPylT_EF1_SMIM38*_Intein_DD & Addgene 162814 \\
\hline L340 & pAS1_4xh7SKPylT_EF1_SMIM38*_(C1A)-Intein_DD & \\
\hline L277.1 & pAS1_4xh7SKPylT_EF1_PIGBOS*_Intein_DD & \\
\hline K090.1 & pAS1_4x7SKPylT_EF1_Ub-*NoBody & Addgene 162807 \\
\hline K047.1 & pAS1_4x7SKPylT_EF1_Ub-*SMIM38 & Addgene 1628008 \\
\hline K067.2 & pAS1_4x7SKPylT_EF1_Ub-*PIGBOS & Addgene 162809 \\
\hline K092.3 & pAS1_4x7SKPylT_EF1_Ub-*Sarcolipin & \\
\hline K099.1 & pAS1_4x7SKPylT_EF1_Ub-*ATP5F1E & \\
\hline K100.2 & pAS1_4x7SKPylT_EF1_Ub-*NDUFB1 & \\
\hline K116 & pAS1_4x7SKPylT_EF1_Ub-*CAV3 & \\
\hline K117 & pAS1_4x7SKPylT_EF1_Ub-*CENPA & \\
\hline K119 & pAS1_4x7SKPylT_EF1_Ub-*VAMP8 & \\
\hline K122.5 & pAS1_4x7SKPylT_EF1_Ub-*CoV2_M & Addgene 162815 \\
\hline K124.1 & pAS1_4x7SKPylT_EF1_Ub-*CoV2_9b & Addgene 162816 \\
\hline K125.2 & pAS1_4x7SKPylT_EF1_Ub-*CoV2_ORF6 & Addgene 162817 \\
\hline K126.1 & pAS1_4x7SKPylT_EF1_Ub-*CoV2_ORF7b & Addgene 162818 \\
\hline
\end{tabular}




\begin{tabular}{|l|l|l|}
\hline K127.2 & pAS1_4x7SKPylT_EF1_Ub-*CoV2_ORF10 & Addgene 162819 \\
\hline K128.2 & pAS1_4x7SKPylT_EF1_Ub-*CoV2_ORF9c/14 & Addgene 162820 \\
\hline K132.2 & pAS1_4x7SKPylT_EF1_Ub-*CoV2_ORF3b_alt & Addgene 162821 \\
\hline K134.10 & pAS1_4x7SKPylT_EF1_Ub-*CoV2_ORF3b(CoV1h) & Addgene 162822 \\
\hline
\end{tabular}

\section{Cell culture, transfection and stable cell line generation}

HEK293T and COS-7 cell lines were maintained in Dulbecco's Modified Eagle Medium (DMEM), containing high glucose, GlutaMAX ${ }^{\mathrm{TM}}$ and pyruvate (Gibco). Medium was supplemented with 10\% fetal bovine serum (FBS, Sigma-Aldrich). All cell lines were cultured in an ambient-controlled incubator at $37^{\circ} \mathrm{C}, 5 \% \mathrm{O}_{2}$ and $5 \% \mathrm{CO}_{2}$. HEK293T cells were forward transfected using TransIT ${ }^{\circledR}$-LT1 (Mirus Bio) according to manufacturer's instructions. COS-7 cells were forward transfected using Lipofectamine LTX $^{\mathrm{TM}}$ with PLUS ${ }^{\mathrm{TM}}$ reagent (Invitrogen) according to manufacturer's protocol. ncAAs were added at the time of transfection as indicated and cells were harvested after 24 or 48 hours. To generate cell lines that stably express the AF variant of the Methanosarcina mazei pyrrolysyl-tRNA synthetase (PylRS-AF), cells were co-transfected with the plasmid E407 and the Super PiggyBac Transposase expression plasmid (System Biosciences). 48 hours post-transfection, cells were selected for at least 7 days with $8 \mu \mathrm{g} / \mathrm{ml}$ (COS-7) or $10 \mu \mathrm{g} / \mathrm{ml}$ (HEK293T) puromycin (Cayman Chemical). Stable cell lines were verified by western blotting or immunofluorescence.

\section{Noncanonical Amino Acids}

All the experiments presented in this study were performed with axial trans-cyclooct-2-ene-1-lysine (TCO*K). TCO*K (SiChem, SC-8008) stock solution was prepared at $100 \mathrm{mM}$ in $0.2 \mathrm{M} \mathrm{NaOH} / \mathrm{H} 2 \mathrm{O}$, $15 \%$ DMSO. To incorporate the ncAA into proteins, working solutions were prepared from the $100 \mathrm{mM}$ stock in the appropriate growth medium and added to the cultured cells together with the transfection mix.

\section{$\underline{\text { SPIEDAC dyes }}$}

Tetrazine-Silicon Rhodamine (tet-SiR, Spirochrome) and 6-Methyl-Tetrazine-BODIPY ${ }^{\circledR}$-FL (me-tet-BDP-FL, Jena Bioscience) stocks were prepared in DMF and further diluted in either RIPA buffer (lysate labeling), TBS-T (fixed cells labeling) or the appropriate growth medium (live cell labeling). 


\section{$\underline{\text { Immunofluorescence and live cell imaging }}$}

For immunofluorescence, cells were grown and transfected in 96-well $\mu$-Plates (ibidi). After withdrawing the ncAA for 4 hours, cells were fixed in $4 \%$ formaldehyde for $10 \mathrm{~min}$ at room temperature and permeabilized for $15 \mathrm{~min}$ with $0.1 \%(\mathrm{v} / \mathrm{v})$ triton/PBS. Prior to incubation with the appropriate antibodies, cells were click-labeled with $500 \mathrm{nM}$ 6-Methyl-tetrazine-BODIPY-FL (Jena Bioscience), washed 3 times with PBS and then blocked for 1 hour in 2\% BSA in TBS supplemented with $0.1 \%$ Tween-20 (TBS-T). Cells were incubated with the HA-probe antibody (F-7, Santa Cruz Biotechnology) overnight at $4^{\circ} \mathrm{C}$. After washing with TBS-T, cells were stained with Alexa555-conjugated secondary antibodies (Life Technologies) for $60 \mathrm{~min}$ at room temperature and counterstained with $1 \mathrm{mg} / \mathrm{ml}$ DAPI (Sigma-Aldrich). After washing, cells were imaged on a Zeiss LSM780 confocal laser scanning microscope using a 40x/1.3 oil objective. Images were processed and prepared for publication using Fiji. $^{2}$

For live cell imaging, cells were grown and transfected in 96-well imaging plates (BD Falcon). After withdrawing ncAA for 1 hour, cells were incubated 30 minutes at $37^{\circ} \mathrm{C}$ in presence of $500 \mathrm{nM}$ me-tet-BDP-FL. Where stated, cells were co-stained with either $4 \mu \mathrm{M}$ Hoechst, $10 \mathrm{mM}$ ER-tracker ${ }^{\mathrm{TM}}$ Red or $250 \mathrm{nM}$ MitoTracker ${ }^{\mathrm{TM}}$ Orange CMTMRos (Invitrogen). After washing 2 times with PBS, cells were immediately imaged in Live Cell imaging Solution (Molecular Probes), on a Nikon eclipse Ti2 inverted widefield microscope equipped with a heated imaging chamber. Images were acquired using a $20 \times / 0.75$ air objective or a 40x/1.15 water objective. For the long-term imaging experiment shown in Supplementary Figure 3, cells were maintained in Leibovitz's L-15 Medium (Gibco) and imaged using a 10x/0.45 air objective. Post-acquisition, images were quantified using CellProfiler. ${ }^{3}$

\section{Protein gels, western blotting and membrane protein extraction}

Cell lysates were prepared in RIPA buffer (50 mM Tris- $\mathrm{HCl}, \mathrm{pH} 7.5,1 \% \mathrm{NP}-40,0.25 \%$ sodium deoxycholate, $150 \mathrm{mM} \mathrm{NaCl}, 1 \mathrm{mM}$ EDTA, and 0.1\% SDS) supplemented with 1x cOmplete ${ }^{\mathrm{TM}}$ Protease inhibitor cocktail. After sonication, the remaining insoluble fraction was removed by centrifugation. Before denaturation, lysates were incubated in presence of $500 \mathrm{nM} \mathrm{SiR}$-tetrazine (Spirochrome) for 10 minutes at RT. Proteins were resolved by SDS-PAGE using 4-20\% Mini-PROTEAN® TGX or $16.5 \%$ Mini-PROTEAN® Tris/Tricine precast polyacrylamide gels (Biorad). SiR-labeled proteins were visualized using an Amersham Imager 600 (GE Healthcare life 
Sciences). If needed, loading was assessed by staining the gels with InstantBlue ${ }^{\mathrm{TM}}$ (Expedeon). Alternatively, proteins were transferred to nitrocellulose membranes (Bio-Rad). Immunoblots were performed using the appropriate primary antibodies and the corresponding HRP-coupled secondary antibodies (Bio-Rad). The following antibodies were employed for western blotting: HA-probe (F-7), GFP (B-2) (Santa Cruz Biotechnology), b-Actin, Calnexin (C5C9), ubiquitin (P4D1) (Cell Signalling) and GAPDH (Merck Millipore). Proteins were visualized on an ImageQuant ${ }^{\mathrm{TM}}$ LAS500 Imager (GE Healthcare Life Sciences) using either the Immobilon Classico or the Immobilon Forte Western HRP substrate (Merck Millipore).

In order to enrich membrane proteins prior to SDS-page, cell pellets were processed with the Mem-PER ${ }^{\mathrm{TM}}$ Plus membrane Protein Extraction Kit (Thermo Scientific) according to manufacturer's instructions.

\section{$\underline{\text { Mass Spectrometry }}$}

HEK293T ${ }^{\text {PylRS-AF }}$ cells were transfected, cultured in the presence of ncAA for 72 hours and lysed in RIPA buffer supplemented with 1x cOmplete protease inhibitor (Roche). The insoluble fraction was removed by centrifugation. Expressed GFP was captured on GFP-Trap_MA magnetic beads (ChromoTEK), washed with RIPA buffer and PBS eluted in $1 \%(\mathrm{v} / \mathrm{v})$ acetic acid.

For intact mass spectrometry, purified GFP samples were desalted and rebuffered into $100 \mathrm{mM}$ ammonium acetate, $\mathrm{pH} 7.5$, using ZebaSpin columns with a $7 \mathrm{kDa}$ cut-off (Thermo Scientific). Samples were directly infused into an Orbitrap Fusion Tribrid mass spectrometer equipped with an offline nanospray source using borosilicate capillaries (Thermo Scientific). The capillary voltage was $1.5 \mathrm{kV}$ and the pressure in the ion-routing multipole was maintained at 0.11 torr. Spectra were acquired in the Orbitrap mass analyzer operated in high mass mode at a resolution of 60.000 between $800-3000 \mathrm{~m} / \mathrm{z}$. Data were analyzed using Excalibur (Thermo Scientific). For tandem mass spectrometry, GFP elution was dried down and resuspended in TEAB. The proteins were purified following the SP3 procedure: ${ }^{4}$ Proteins were reduced and alkylated by incubating with TCEP and CAA respectively. They were bound by the addition of and washed with $\mathrm{EtOH}$. Proteins were digested with trypsin and incubated at $37^{\circ} \mathrm{C}$ for 18 h. Resulting tryptic peptides were analysed on a Q Exactive HF (Thermo Scientific). 
A

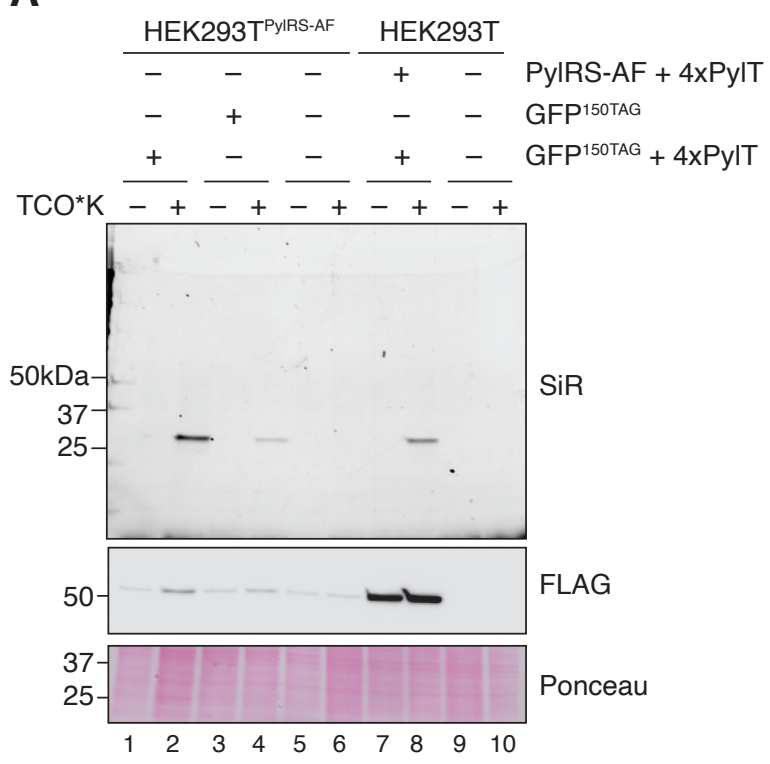

B

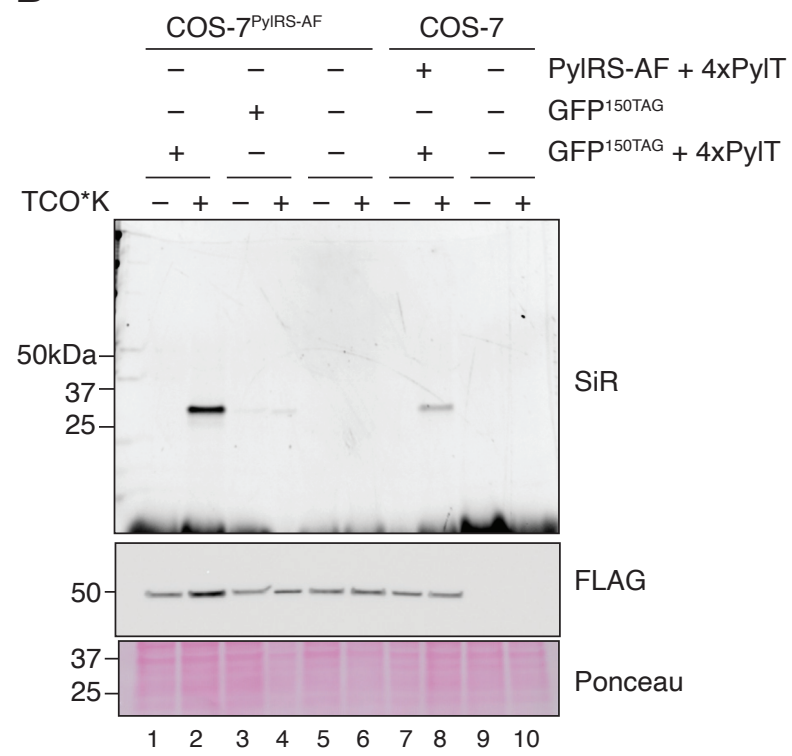


Supplementary Figure 1 - Characterization of transgenic HEK293T and COS-7 cell lines expressing PylRS-AF and 4x PylT. (A) HEK293T cells were transfected with plasmid pAS1_4xh7SKPylT_EF1_PylRSAF_IRES_Puro, bearing FLAG-PylRS-AF and 4 copies of PylT, and PiggyBac transposase to generate the stable cell line HEK293T ${ }^{\text {PyIRS-AF }}$ as described. ${ }^{5}$ Plasmids bearing GFP $^{150 T A G}$ and either none or 4 copies of PylT were transfected into HEK293T ${ }^{\text {PylRS-AF }}$ (lanes 1-6). For comparison, the plasmid bearing PylRS-AF and 4 copies of PylT and the plasmid bearing GFP ${ }^{150 \mathrm{TAG}}$ and 4 copies of PylT were transiently cotransfected at 1:1 stoichiometry into the parental HEK293T cell lines (lanes 7-10). Cells were grown for 48 hours with $50 \mu \mathrm{M} \mathrm{TCO} * \mathrm{~K}$ and lysates were labeled with tet-SiR. After SDS-PAGE, fluorescence was imaged in-gel and PylRS-AF levels were assessed by anti-FLAG western blotting. (B) COS-7 were transfected with plasmid pAS1_4xh7SKPylT_EF1_PylRSAF_IRES_Puro, bearing FLAG-PylRS-AF and 4 copies of PylT, and PiggyBac transposase to generate the stable cell line COS-7 $7^{\mathrm{PyIRS}-\mathrm{AF}}$ as described. ${ }^{5}$ Plasmids bearing $\mathrm{GFP}^{150 \mathrm{TAg}}$ and either none or 4 copies of PylT were transfected into COS-7 $7^{\text {pylRS-AF }}$ (lanes 1-6). For comparison, the plasmid bearing PylRS-AF and 4 copies of PylT and the plasmid bearing GFP ${ }^{150 \mathrm{TAG}}$ and 4 copies of PylT were transiently cotransfected at 1:1 stoichiometry into the parental COS-7 cell lines (lanes 7-10). Cells were grown for 48 hours with $50 \mu \mathrm{M}$ TCO*K and lysates were labeled with tet-SiR. After SDS-PAGE, fluorescence was imaged in-gel and PylRS-AF levels were assessed by anti-FLAG western blotting. Stably integrated PylRS-AF was efficient in suppressing the GFP ${ }^{150 T A G}$ even if expressed at considerably lower levels in HEK293 TyIRS-AF $^{\text {cells. }}$ 


\section{Supplementary Figure 2 -}

Names and chemical structures of the ncAA and SPIEDAC dyes used in this study.

TCO*K

trans-cyclooct-2-ene--Iysine

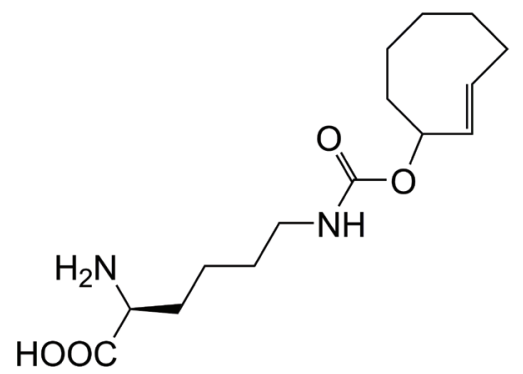

me-tet-BDP-FL

6-Methyl-Tetrazine-BODIPY®-FL

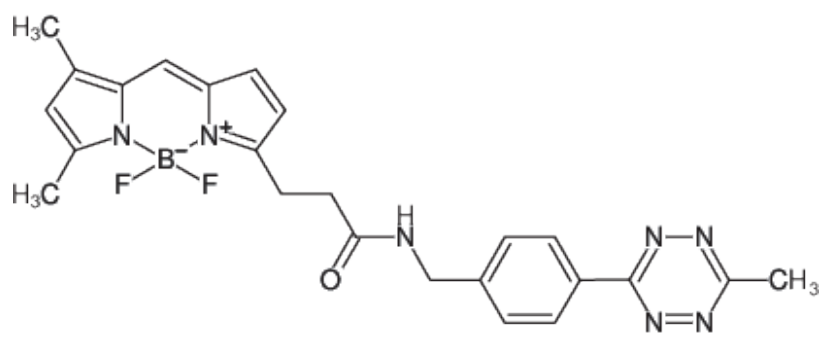

tet-SiR

tetrazine-Silicon Rhodamine<smiles>CN(C)c1ccc2c(c1)[Si](C)(C)c1cc(N(C)C)ccc1C21OC(=O)c2ccc(C(=O)NCc3ccc(-c4nncnn4)cc3)cc21</smiles> 
Supplementary Figure 2 - Names and chemical structures of the ncAA and SPIEDAC dyes used in this study. 


\section{Supplementary Figure 3 - Mass Spectrometry of N-terminally labeled GFP and NoBody}

A

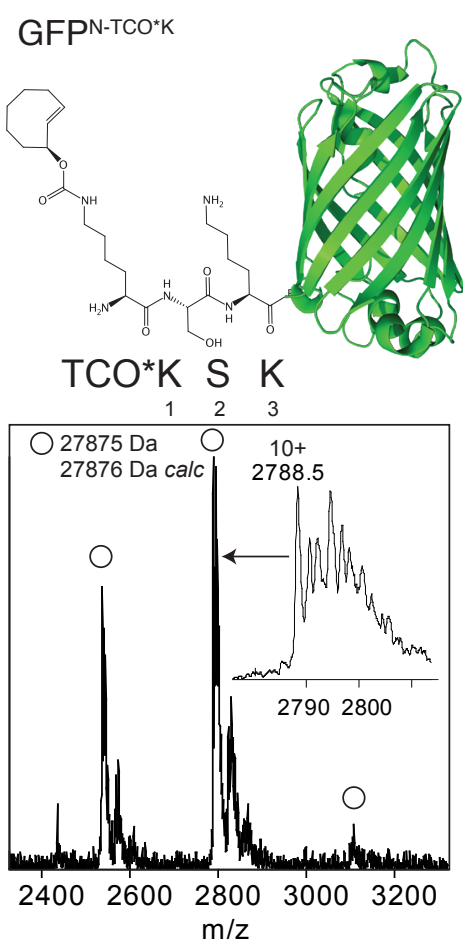

B

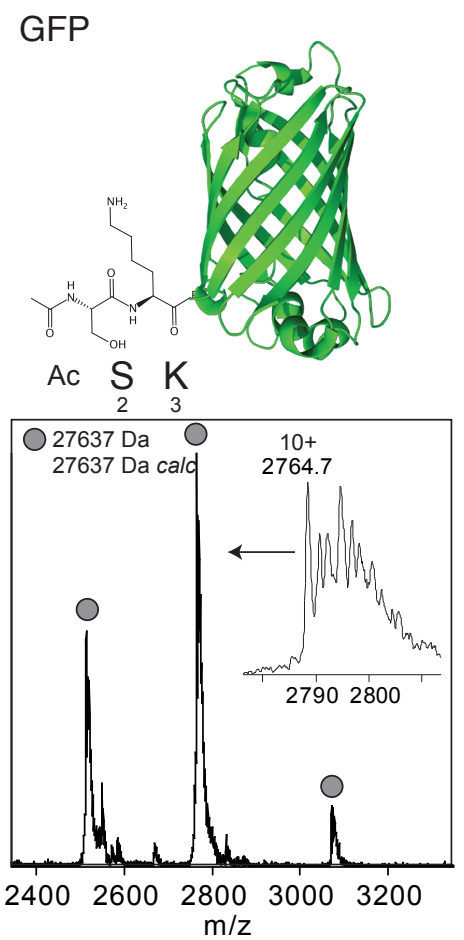

Construct
GFP
GFP N-TAG

GFP N-TAG

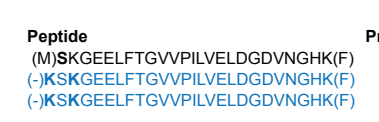

$\begin{array}{lcr}\text { Probability lon } \\ \text { Pro } \\ \text { F) } & 100 \% & 137.3 \\ \text { F) } & 100 \% & 49.83 \\ & 95 \% & 16.2\end{array}$
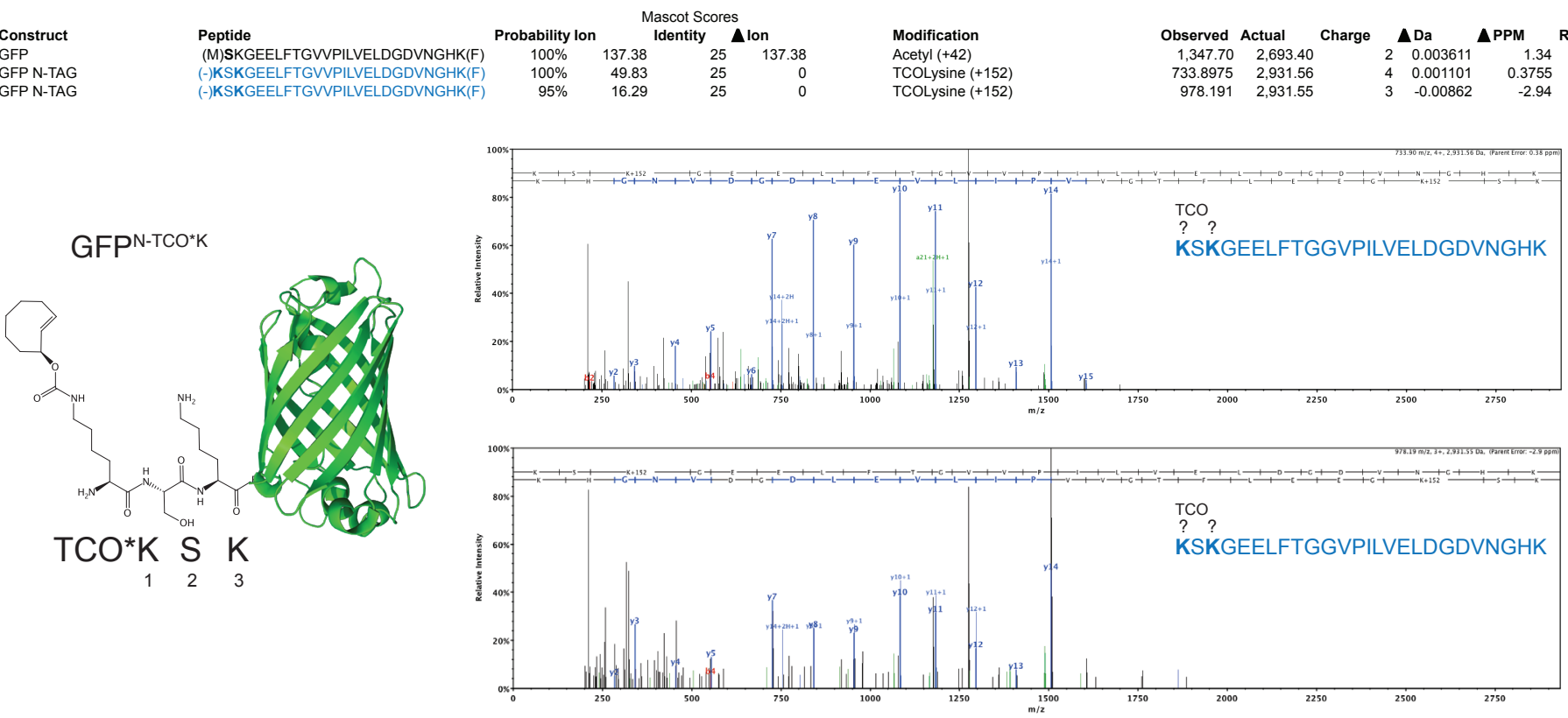

D

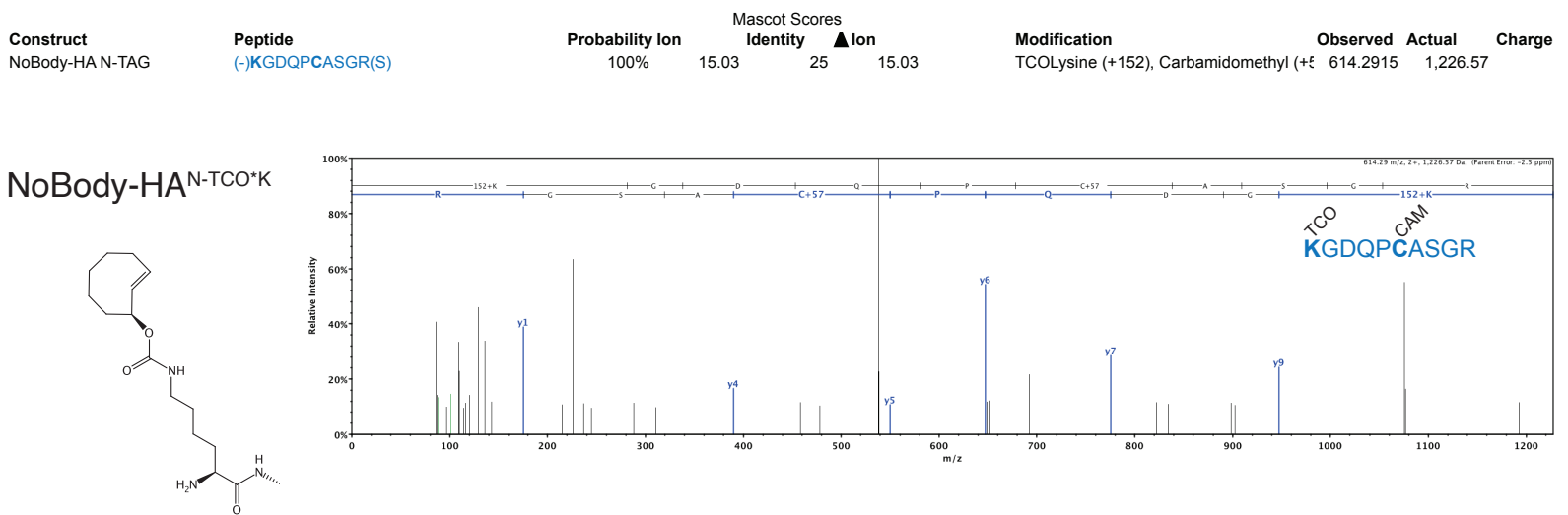

TCO*KGDQPCASGRSTLPPGNAREAKPPKKRCLLAPRWDYPEGTPNGGSTTLPSAPPPASAGLKSHPPPPEKYPYDVPDYA 
Supplementary Figure 3 - Mass Spectrometry of N-terminally labeled GFP and NoBody. (A) Intact Mass determined for GFP expressed in HEK293 $\mathrm{T}^{\mathrm{PyIR}-\mathrm{AF}}$ from Ub-GFP ${ }^{\mathrm{N}-\mathrm{TAG}}$ in the presence of TCO*K confirms that deubiquitinases cleave off ubiquitin $\mathrm{N}$-terminal of $\mathrm{TCO}^{*} \mathrm{~K}$, yielding $\mathrm{GFP}^{\mathrm{N}-\mathrm{TCO}^{*} \mathrm{~K}}$ with a free (non-acetylated) N-terminus. (B) As a control, intact mass was also determined for native GFP

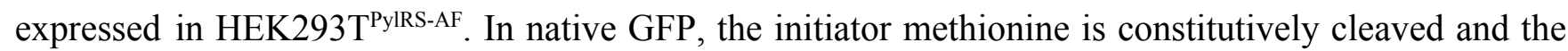
new N-terminus is subsequently acetylated. Thus, $\mathrm{GFP}^{\mathrm{N}-\mathrm{TCO}^{* \mathrm{~K}}}$ has an additional mass of $238 \mathrm{Da}$ as compared to native GFP (free TCO*K 298 Da versus acetic acid $60 \mathrm{Da}$ ). (C) Tandem mass spectrometry of GFP ${ }^{\mathrm{N}-\mathrm{TCO} * \mathrm{~K}}$ tryptic peptides. Detection of the N-terminus was only possible in a subset of peptides where K3 was not cleaved by trypsin, yielding a 25-amino acid peptide in GFP (2693.40 Da) and a 26-amino acid peptide in $\mathrm{GFP}^{\mathrm{N}-\mathrm{TCO}^{* \mathrm{~K}}}$ (2931.56). An exact mass difference of $238 \mathrm{Da}$ was observed between $\mathrm{GFP}^{\mathrm{N}-\mathrm{TCO} * \mathrm{~K}}$ native GFP N-terminal peptides. MS/MS spectrum did not allow discerning if the 152 adduct corresponding to $\mathrm{TCO} * \mathrm{~K}$ was located on $\mathrm{K} 1$ or $\mathrm{K} 3$ due to the lack of $b$ fragments. (D) Tandem mass spectrometry of NoBody ${ }^{\mathrm{N}-\mathrm{TCO}^{*} \mathrm{~K}}-\mathrm{HA}$ tryptic peptides. The N-terminal tryptic 10-amino acid peptide could be unequivocally assigned, carrying the specific 152 Da mass adduct for TCO* on $\mathrm{K} 1$ in addition to the constitutive thiol alkylation on $\mathrm{C} 6$. 
Supplementary Figure 4 -

Quantification and comparison of Ub-GFP ${ }^{150 T A G}$ and Ub-GFP ${ }^{\mathrm{N}-\mathrm{TAG}}$ expression kinetics.
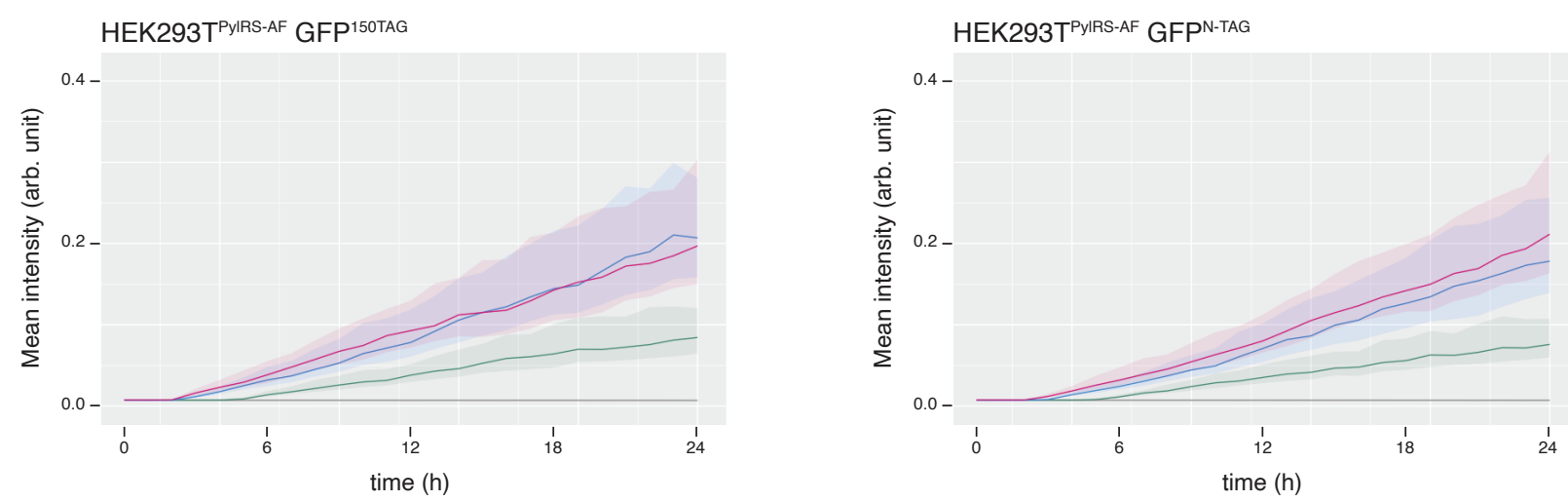

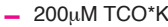

- $50 \mu \mathrm{M}$ TCO*K

- $10 \mu \mathrm{M} \mathrm{TCO} \mathrm{K}^{*}$

- untr.
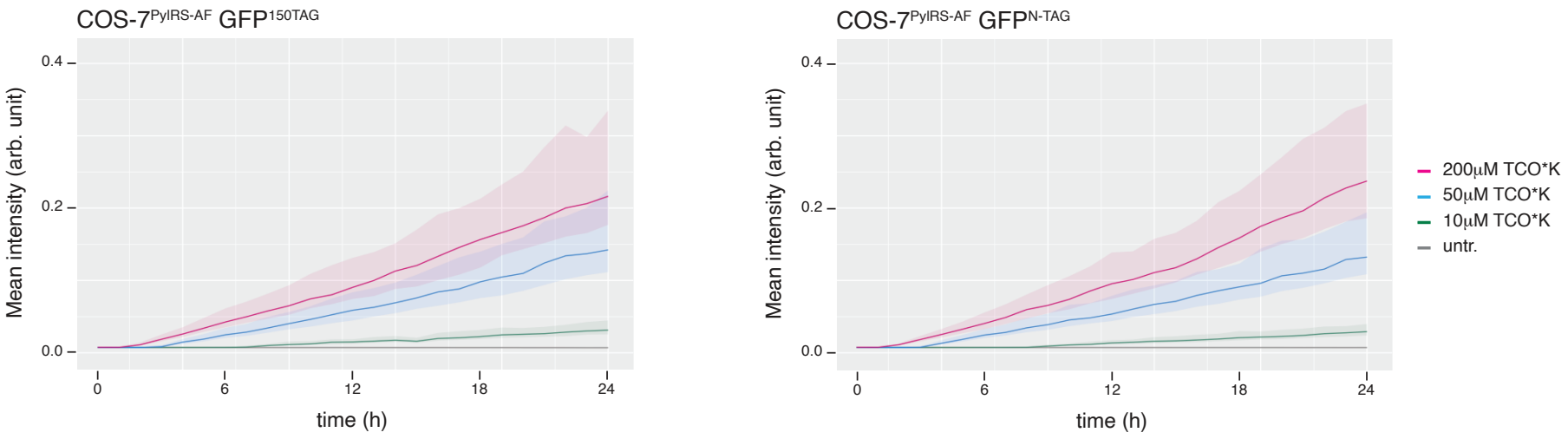
Supplementary Figure 4 - Quantification and comparison of $\mathrm{GFP}^{150 \mathrm{TAG}}$ and $\mathrm{GFP}^{\mathrm{N}-\mathrm{TAG}}$ expression kinetics. Plasmids expressing Ub-GFP ${ }^{150 \mathrm{TAG}}$ or $\mathrm{Ub}-\mathrm{GFP}^{\mathrm{N}-\mathrm{TAG}}$ and 4 copies of PylT were transfected into HEK293T ${ }^{\text {PylRS-AF }}$ or COS-7 ${ }^{\text {PylRS-AF }}$ cells. 24 hours after transfection, indicated final concentrations of TCO*K were added and cells were imaged in 1 hour intervals using a Nikon eclipse Ti2 inverted widefield microscope for 24 hours. Post-acquisition, images were quantified using CellProfiler. ${ }^{3}$ Plotted are the median, first quartile and third quartile values that were calculated using the mean GFP intensity of 500 GFP-positive cells. Overall, GFP ${ }^{150 \mathrm{TAG}}$ and GFP ${ }^{\mathrm{N}-\mathrm{TAG}}$ showed comparable kinetics in both cell lines at all tested $\mathrm{TCO}^{*} \mathrm{~K}$ concentrations. 


\section{Supplementary Figure 5 -}

\section{$\mathrm{C}$-terminal single residue labeling tag.}

A

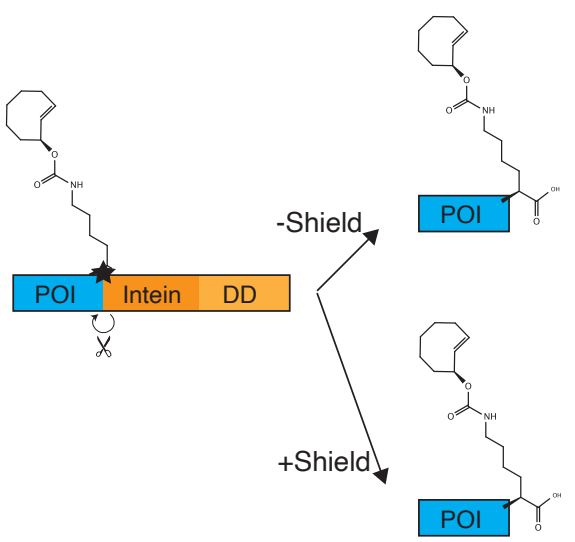

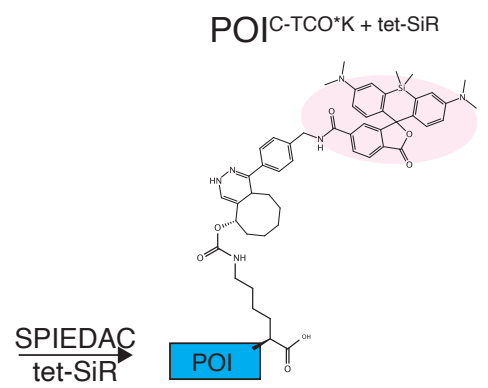

B

GFP

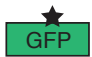

GFP Intein DD

GFP AIntein DD

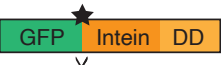

GFP AIntein DD

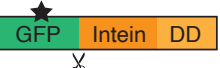

$\mathrm{kDa}: \frac{28 \frac{18 \quad 12}{30}}{58}$
C

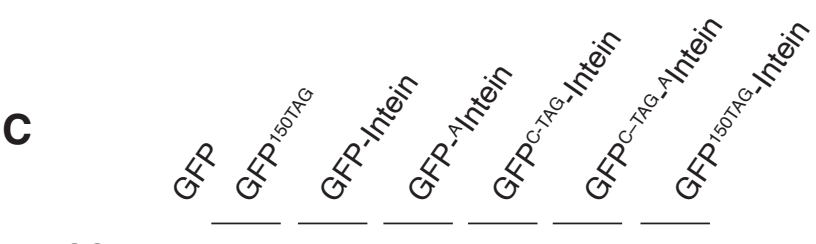

$$
\text { TCO*K - - + - - - + + + - + }
$$

Shield-1 - - - + +++-+++ 100
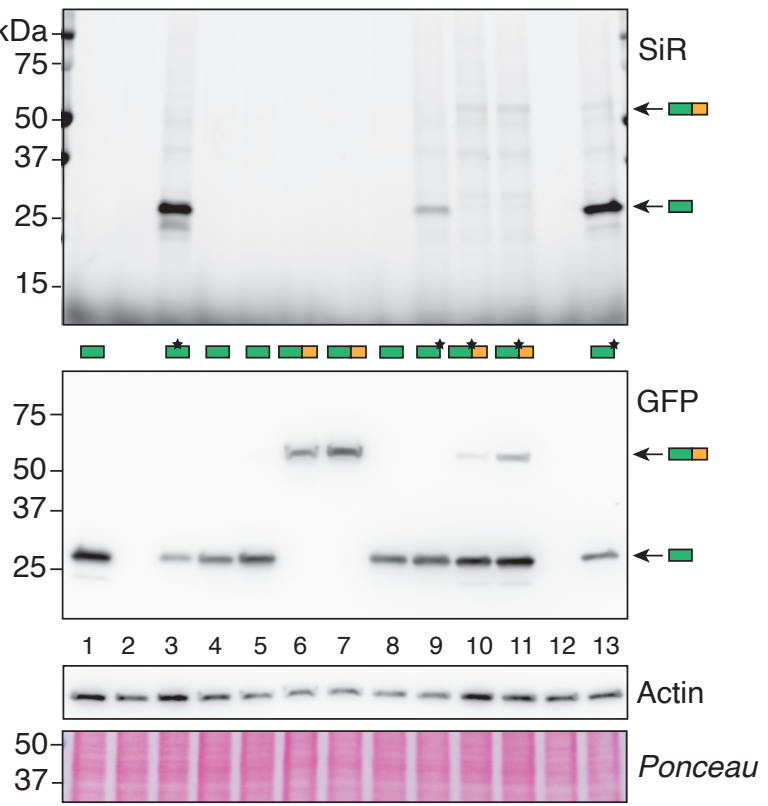
Supplementary Figure 5 - C-terminal single residue labeling tag. (A) Construct to incorporate ncAAs at the C-terminus of a protein of interest (POI). A self-cleaving mini-intein is positioned after the relevant coding sequence, with its endogenous stop codon being replaced by an amber stop codon. When the amber codon is suppressed, the microprotein-intein fusion is produced as an intermediate product, from which a microprotein bearing a C-terminal ncAA is released. A degradation domain (DD) is C-terminally fused to limit the accumulation of processed and incompletely-processed byproducts. (B) Summary of the intein-based constructs tested in panel B. In this pilot set of constructs, GFP serves as a proof-of-principle protein of interest. The position of the ncAA is indicated as a star. (C) The different constructs presented in panel A were transiently expressed in HEK293T ${ }^{\text {PylRS-AF }}$ and the medium simultaneously supplemented with $50 \mu \mathrm{M}$ TCO*K. 24 hours after transfection, cells were lysed in RIPA buffer. Thereafter, lysates were labeled with tet-SIR and analyzed by western blot. Overexpression of native GFP in the context of the intein system resulted in a single band of the expected size of GFP (lane 4). This result indicates that GFP is excised from the intein domain in living cells. The level of GFP is increased when cells are treated with Shield-1, an inhibitor of the degradation domain, ${ }^{6}$ suggesting that, to some extent, initiation of protein degradation preceded intein cleavage (compare lane 4 to lane 5). Importantly, the intein cleavage was still efficient for the GFP ${ }^{\mathrm{C}-\mathrm{TAG}}$ variant, where the splice site Cys was immediately preceded by $\mathrm{TCO}^{*} \mathrm{~K}$. As in the case of the experiments using the ubiquitin-based constructs, the GFP variants carrying TCO*K could be readily detected by in-gel labeling (lanes 3, 9 and 13). Finally, when the catalytic activity of the intein was abrogated, the GFP-Intein fusion but not the cleaved GFP product could be detected by in-gel labeling, suggesting that the fusion protein remained unprocessed as expected (Figure 3B, lanes 6, 7 and 10,11). However, GFP signal corresponding to non-fusion and non-ncAA tagged GFP could still be observed by western blotting (Figure 3B, lanes 10 and 1). 
Supplementary Figure 6 -

STELLA N- and C-terminal processing controls.

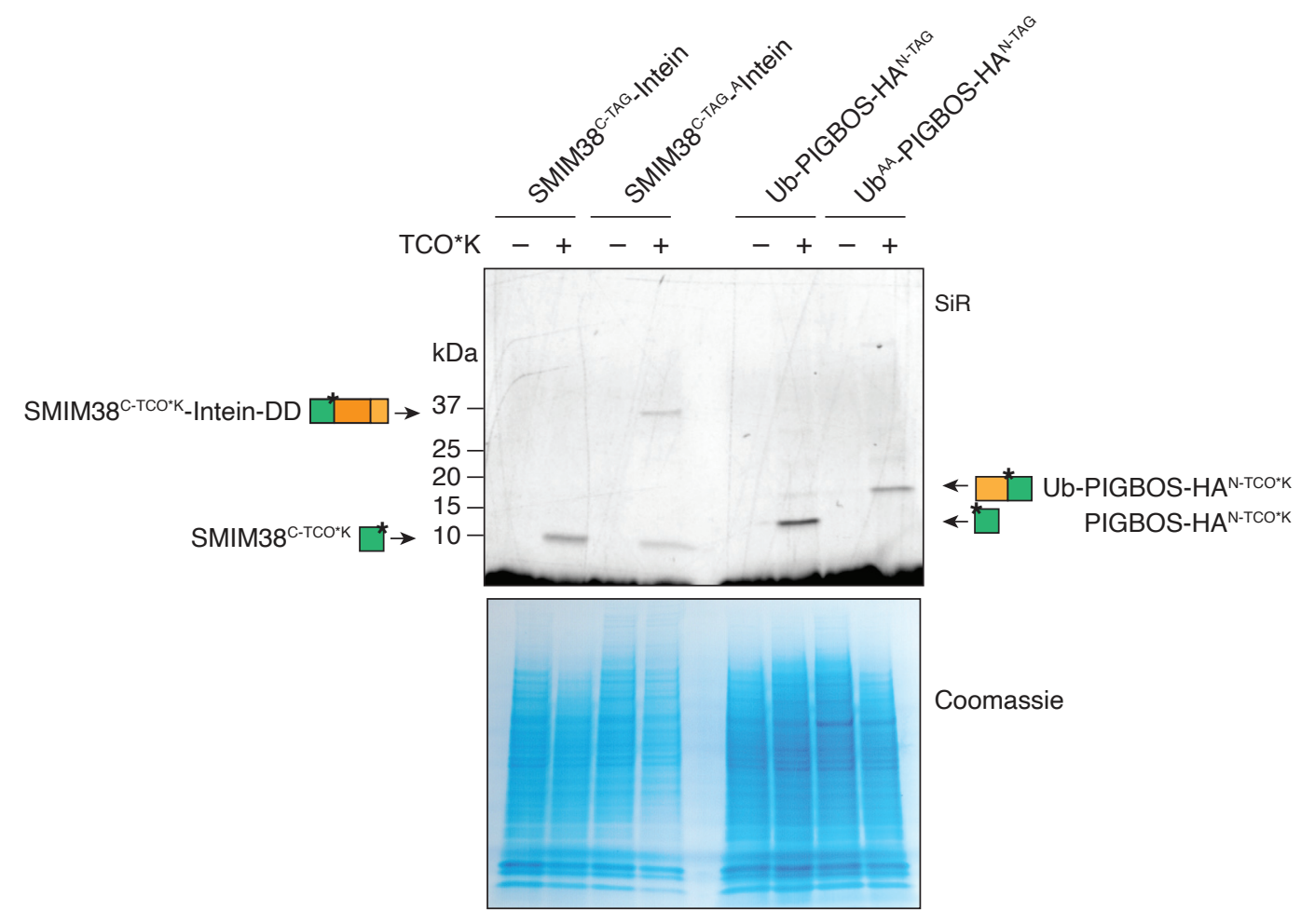


Supplementary Figure 6 - STELLA N- and C-terminal processing controls. Constructs carrying PIGBOS $^{\text {N-TAG }}-\mathrm{HA}$, SMIM38 $8^{\mathrm{C}-\mathrm{TAG}}$ or their respective processing-dead version were transiently transfected into HEK293T $\mathrm{T}^{\text {PylRS-AF }}$. Cells were incubated for 48 hours with $50 \mu \mathrm{M}$ TCO*K. Lysates were labeled with tet-SiR and after SDS-PAGE analyzed by in-gel fluorescence imaging and Coomassie staining. 
Supplementary Figure 7 -

STELLA N- and C-terminal labeling of GFP and SMIM38 in three mammalian cell lines.

A

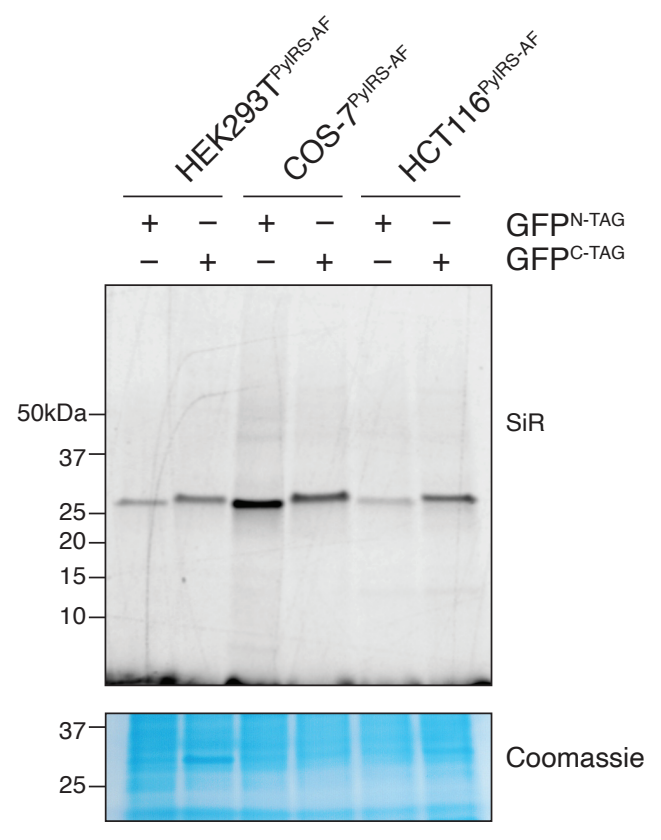

B

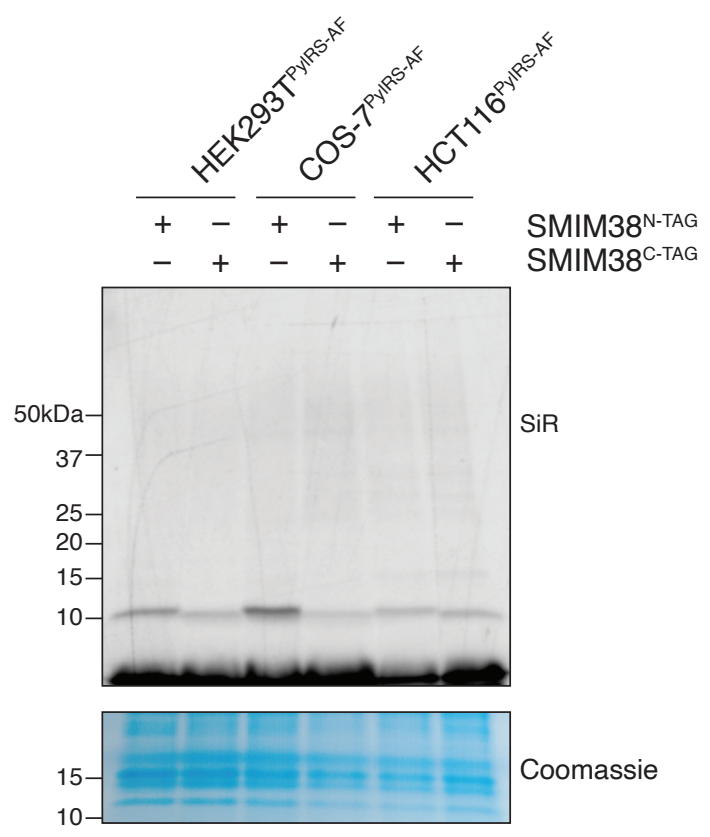


Supplementary Figure 7 - STELLA N- and C-terminal labeling of GFP and SMIM38 in three mammalian cell lines. The indicated constructs $\mathrm{GFP}^{\mathrm{N}-\mathrm{TAG}}, \mathrm{GFP}^{\mathrm{C}-\mathrm{TAG}}(\mathrm{A}), \mathrm{SMIM}^{\mathrm{N}-\mathrm{TAG}}$ or SMIM38 $8^{\mathrm{C}-\mathrm{TAG}}$ (B) were transfected into HEK293T $\mathrm{T}^{\text {PylRS-AF }}$, COS- $7^{\text {PylRS-AF }}$ or HCT116 $6^{\text {PylRS-AF }}$. Cells were incubated for 48 hours with $50 \mu \mathrm{M}$ TCO*K. Lysates were labeled with tet-SiR and after SDS-PAGE analyzed by in-gel fluorescence imaging and Coomassie staining. 
Supplementary Figure 8 -

Comparison of SPIEDAC labeling with two different cell-permeable dyes.

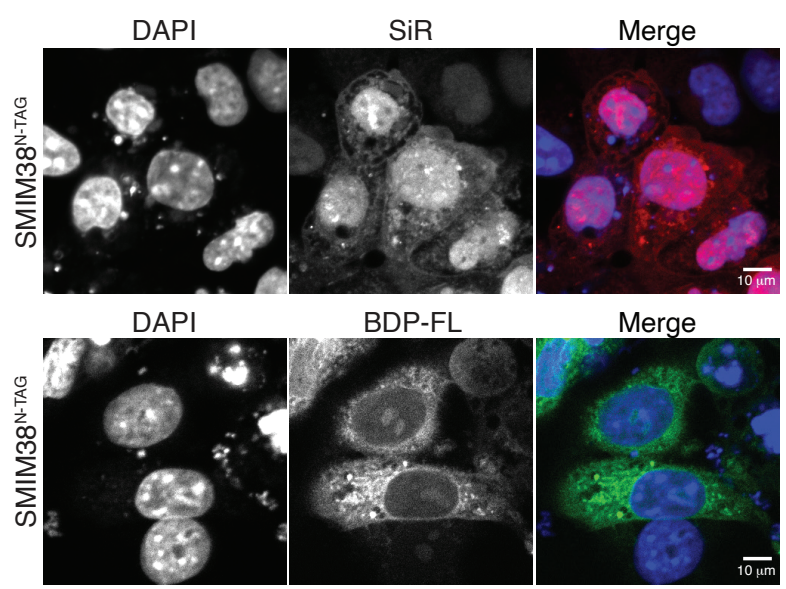


Supplementary Figure 8 - Comparison of SPIEDAC labeling with two different cell-permeable dyes. COS- $7^{\text {PyIRS-AF }}$ were transfected with SMIM $38^{\text {N-TAG }}$ and incubated for 24 hours with TCO*K. The ncAA-containing protein was then reacted with either tet-SiR or me-tet-BDP-FL fluorescent dyes in fixed and permeabilized cells. Cells were imaged by confocal microscopy. 


\section{Supplementary Figure 9-}

SPIEDAC labeling controls experiments on fixed cells.

A

Transfected (SMIM38 $\left.{ }^{\mathrm{N}-\mathrm{TAG}}-\mathrm{HA}\right)$

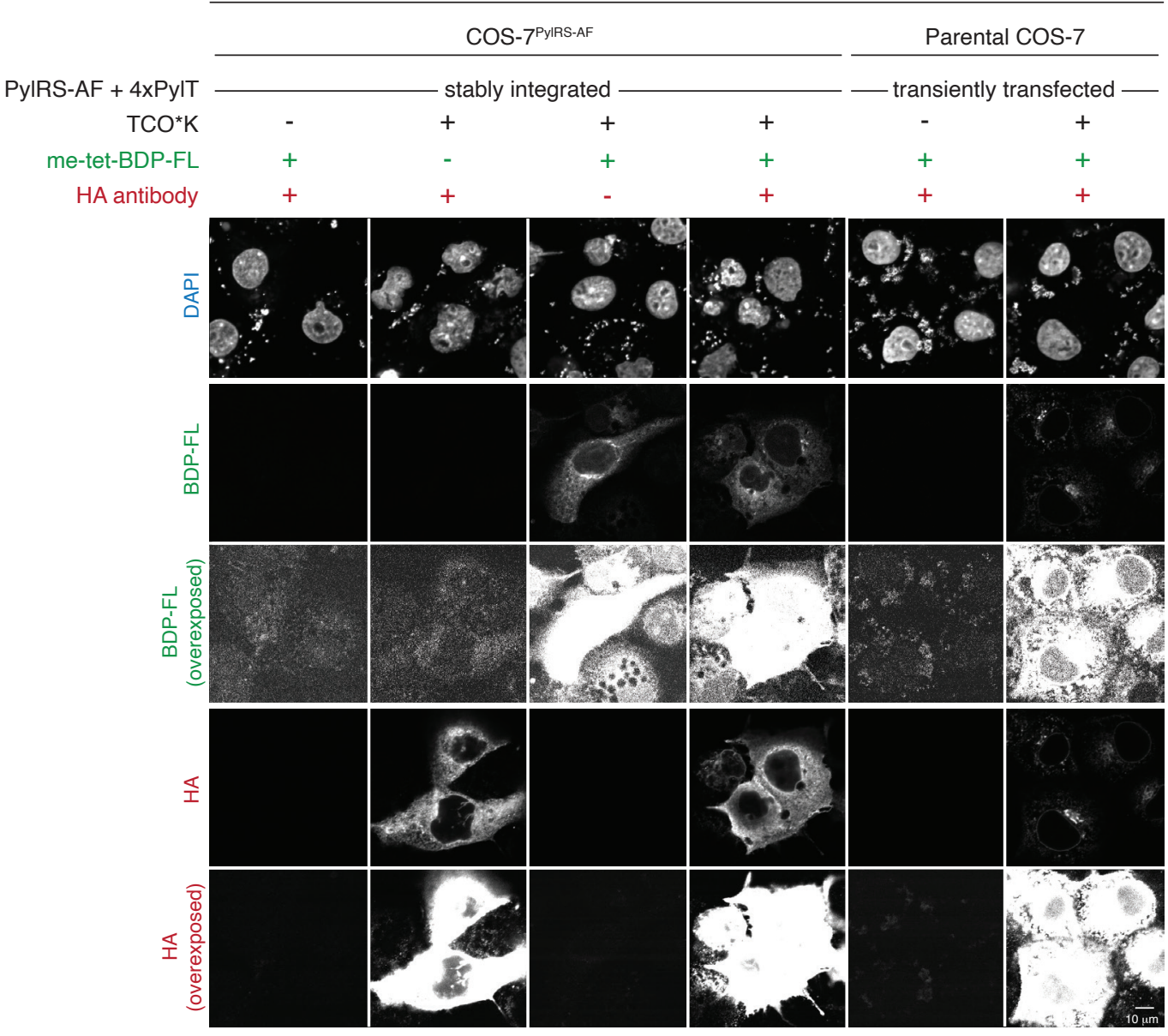

B
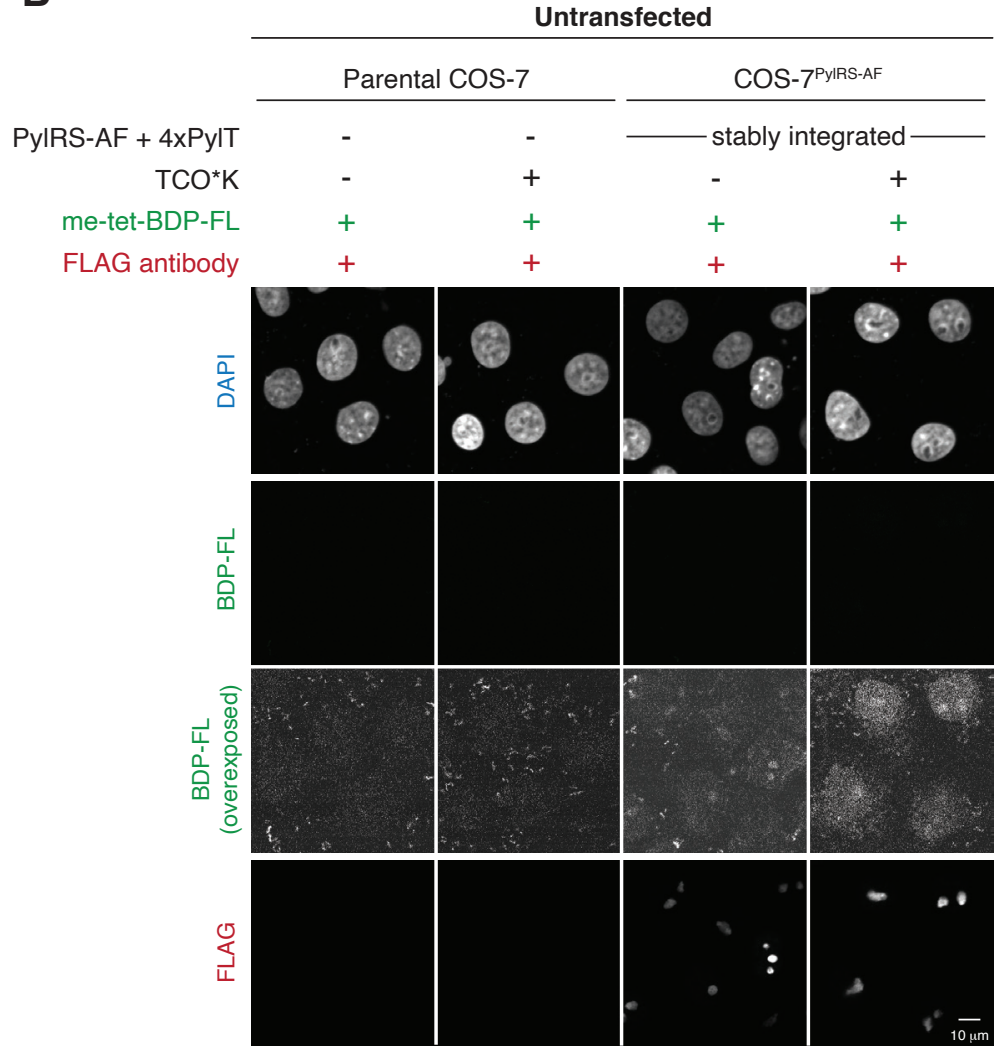

C

HEK293T PyIRS-AF

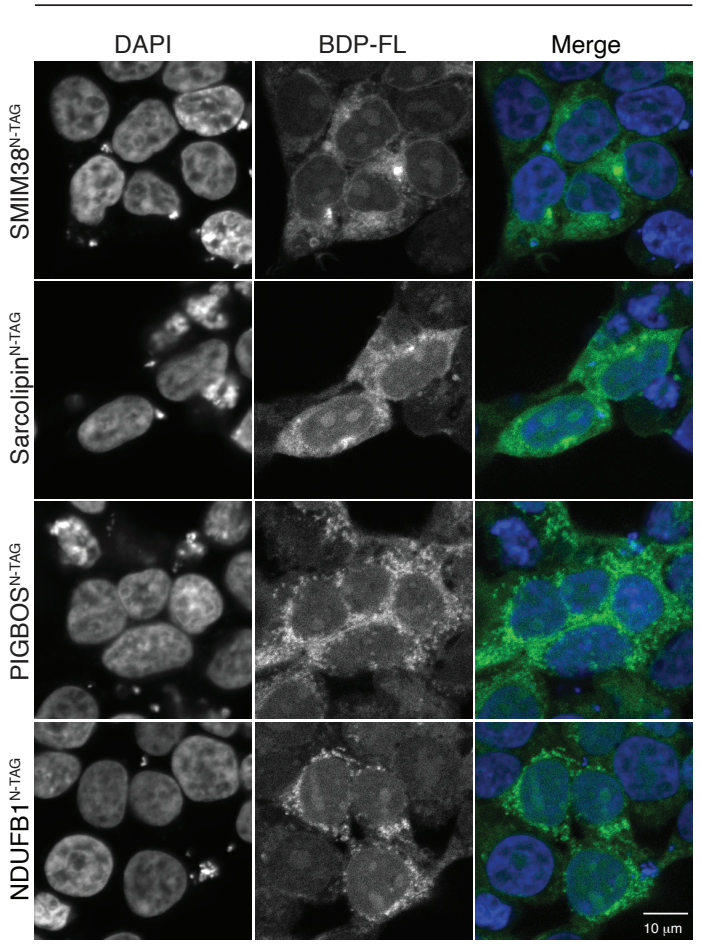


Supplementary Figure 9 - SPIEDAC labeling control experiments on fixed cells. (A) COS-7 $7^{\text {PyIRS-AF }}$ or COS-7 cells were transfected with the SMIM38 ${ }^{\mathrm{N}-\mathrm{TAG}}-\mathrm{HA}$ construct and treated as described in Figure 2. Reagents (TCO*K, met-tet-BDP-FL, anti-HA antibody) were left out as indicated and staining was compared to positive controls (columns 4, 6). (B) COS-7 $7^{\text {PylRS-AF }}$ or COS-7 cells were not transfected but treated as described in Figure 2. Staining with anti-FLAG antibody highlights the localization of PylRS-AF. Image acquisition settings were fixed for all images of panels A and B. (C) HEK293T ${ }^{\text {PylRS-AF }}$ were transfected with the indicated constructs and incubated for 24 hours with $50 \mu \mathrm{M}$ TCO*K. Cells were subsequently fixed, permeabilized and labeled with met-tet-BDP-FL. 
Supplementary Figure 10 -

Colocalization of BDP-FL and HA signals in confocal images

A Background subtraction (rolling ball, 50px) Li thresholding

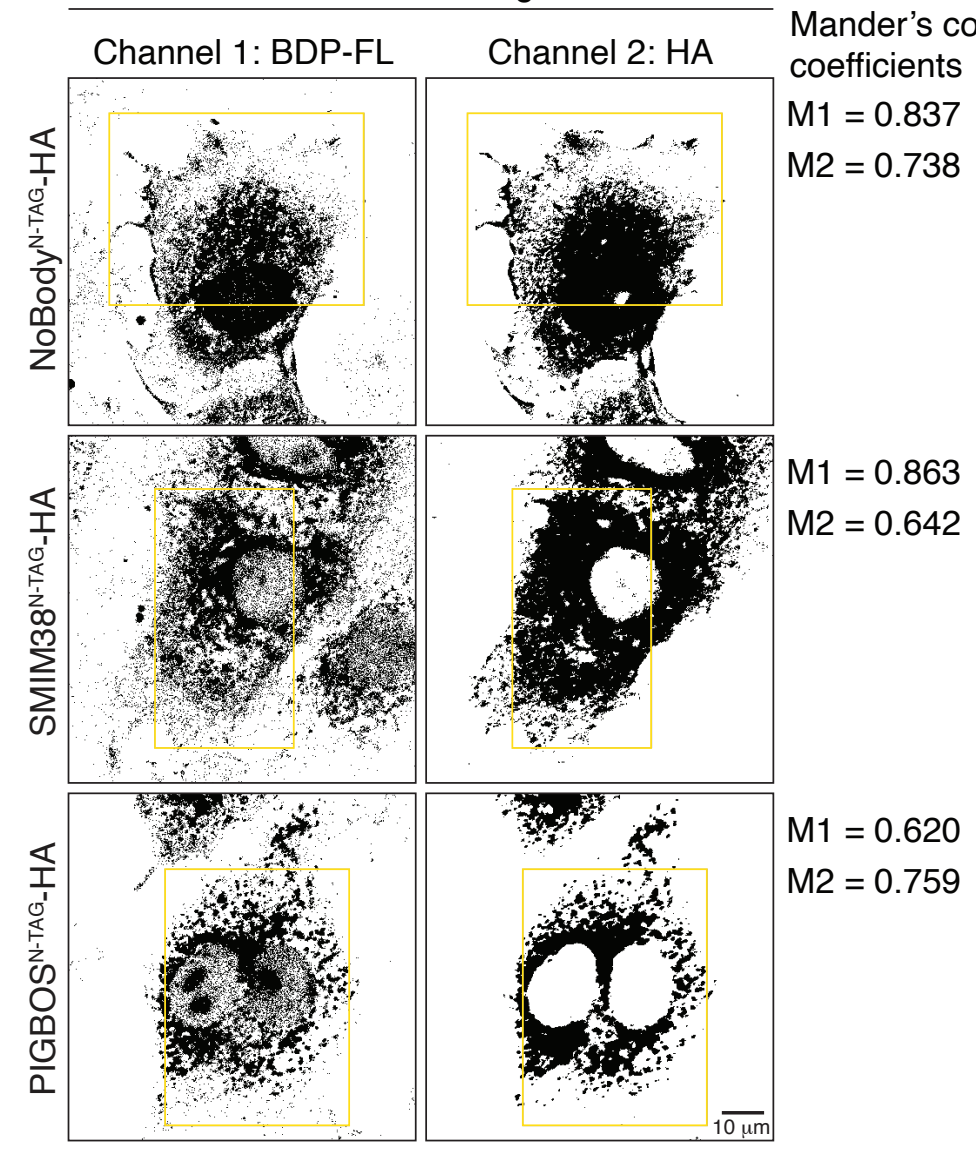

B

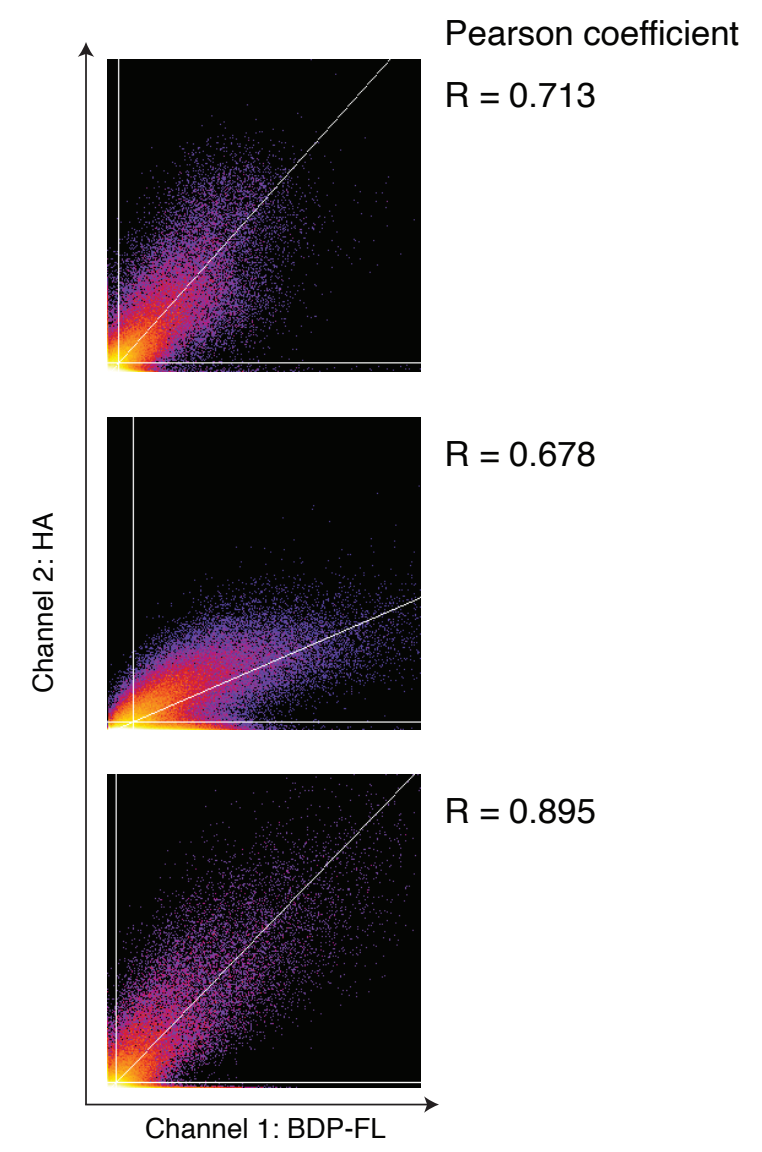


Supplementary Figure 10 - Calculation of colocalization coefficients . (A) The confocal images shown in Figure 2B are presented after background subtraction (rolling ball, 50 pixels) and Li's minimum cross entropy thresholding. ${ }^{7}$ The binomial images were used to calculate Mander's split coefficients ${ }^{8}$ (M1 and M2) for each pair of channels (channel 1 being BDP-FL and channel 2 being HA) within the highlighted region of interest (ROI). (B) Scatter plots of the pixel intensities of the BDP-FL and HA channels of the images shown in Figure 2. The Pearson's correlation coefficient for pixels above the Costes' automatic threshold ${ }^{9}$ is presented on the right side of each plot. The same ROIs as shown in (A) were used for quantification. Images were processed and analyzed using Fiji. ${ }^{2}$ 
Supplementary Figure 11 -

Live cell fluorescent labeling using STELLA N-terminal tag and SPIEDAC.

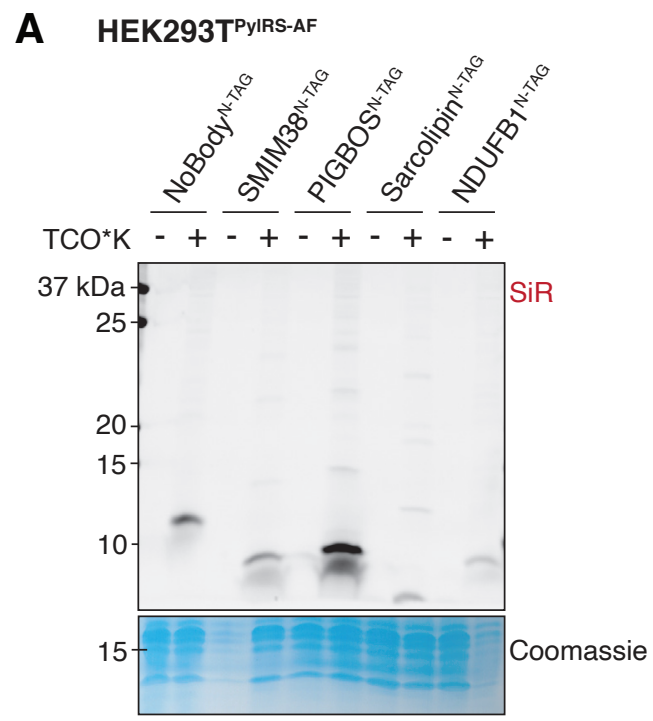

B $\cos -7^{\text {PyIRS-AF }}$

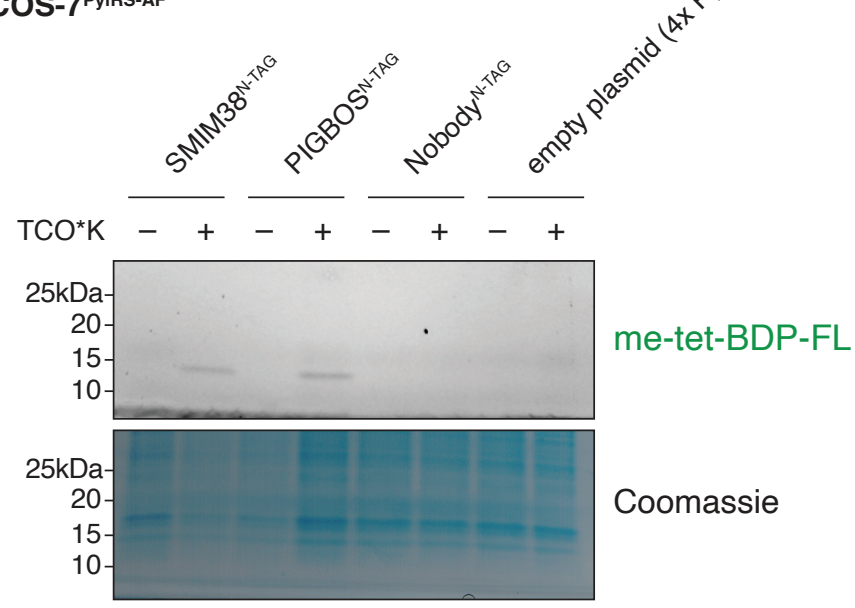

C $\cos -7^{\text {PyIRS-AF }}$

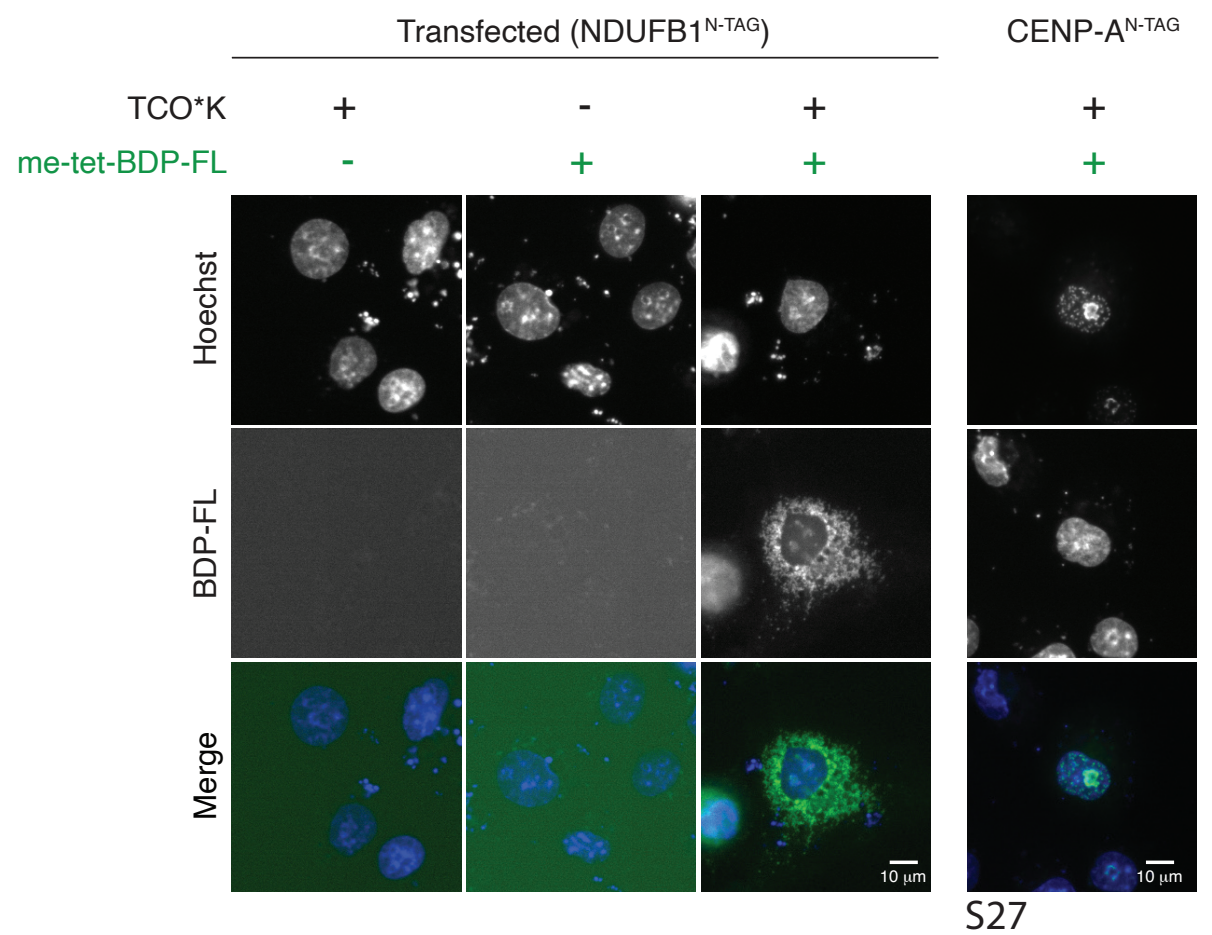


Supplementary Figure 11 - Live cell fluorescent labeling using STELLA N-terminal tag and SPIEDAC. (A) HEK293T ${ }^{\text {PylRS-AF }}$ were transfected with the indicated constructs and incubated for 48 hours with $50 \mu \mathrm{M}$ TCO*K. Cells were subsequently labeled with tet-SiR, washed, lysed and analyzed by SDS-PAGE. (B) Cells were transfected and treated exactly as in Figure 4, but subsequently lysed and analyzed by SDS-PAGE. Because of the $24 \mathrm{~h}$ incubation time used for live cell imaging and higher gel autofluorescence in the green channel, the sensitivity of detecting met-tet-BDP-FL labeled microproteins was limited, and only SMIM $38^{\mathrm{N}-\mathrm{TCO} * \mathrm{~K}}$ and PIGBOS ${ }^{\mathrm{N}-\mathrm{TCO}{ }^{* \mathrm{~K}}}$ but not NoBody ${ }^{\mathrm{N}-\mathrm{TCO} \mathrm{K}^{\mathrm{K}}}$ could be visualized in-gel. Additional microproteins shown in Figure 4 were not tested. (C) Live cell labeling controls treated as in Figure 4, omitting either $\mathrm{TCO} * \mathrm{~K}$ or met-tet-BDP-FL. No significant fluorescence was observed in the negative controls. To control for non-specific cytosolic or nuclear background fluorescence in the presence of both $\mathrm{TCO}^{*} \mathrm{~K}$ and met-tet-BDP-FL, two proteins with defined locations, NDUFB1 (mitochondrial) and CENP-A (nuclear/centromeric) were compared. 
Supplementary Figure 12 -

Labeling of SARS-CoV-2 derived micropeptides in fixed cells.

A $\quad$ COS-7 $7^{\text {PyIRS-AF }}$

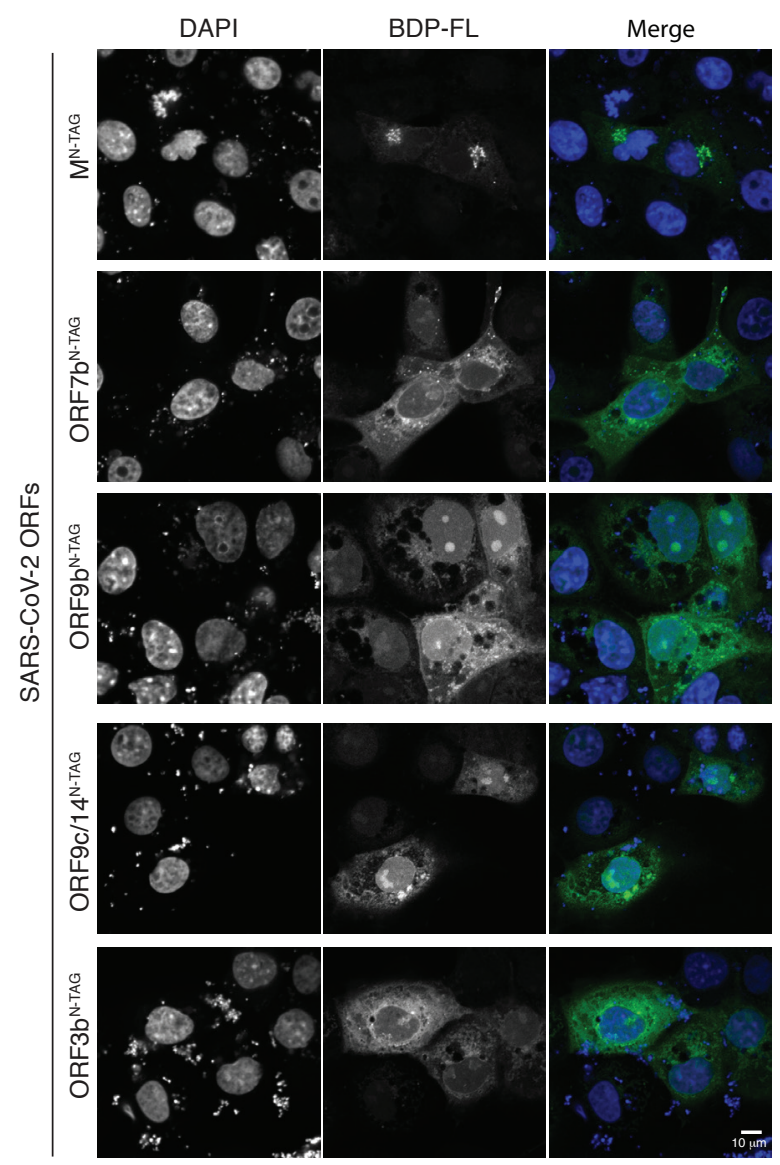

TraDSNGIIIVEELKKLLEO NNLVIGFLFLIWICLIQFAYANRNRFLYIIKLIF 52 53 LWLLWPV LLACFVLAAVYRINWI IG IAIAMACLVGLMWLSYFIASFRLFAR 104 105 IRSMWSFNPEINILLNVPLHGIIIRPLLESELVIGAVILRGHLRIAGHHL 156 157 RCDIKDLPKEITVATSRILSYYKLGASORVAGDSGFAAYSRYRIG NYLNID 208 209 HSSSSDNIALLVO*

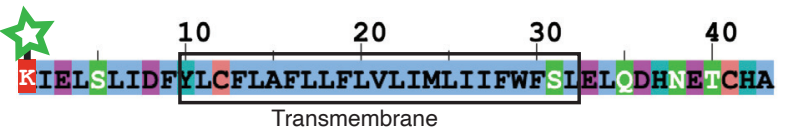

\begin{tabular}{|c|c|c|c|c|}
\hline & 10 & 20 & 30 & 40 \\
\hline 0 & 60 & 70 & 80 & 9 \\
\hline
\end{tabular}

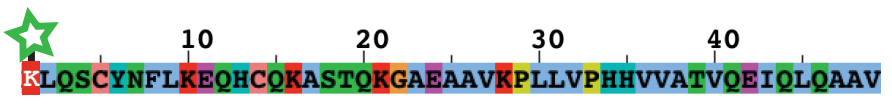
$\begin{array}{lll}50 & 60 & 70\end{array}$ GELLLLEWLAMAVMLLLLCCCLTD

B HEK293T PyIRS-AF

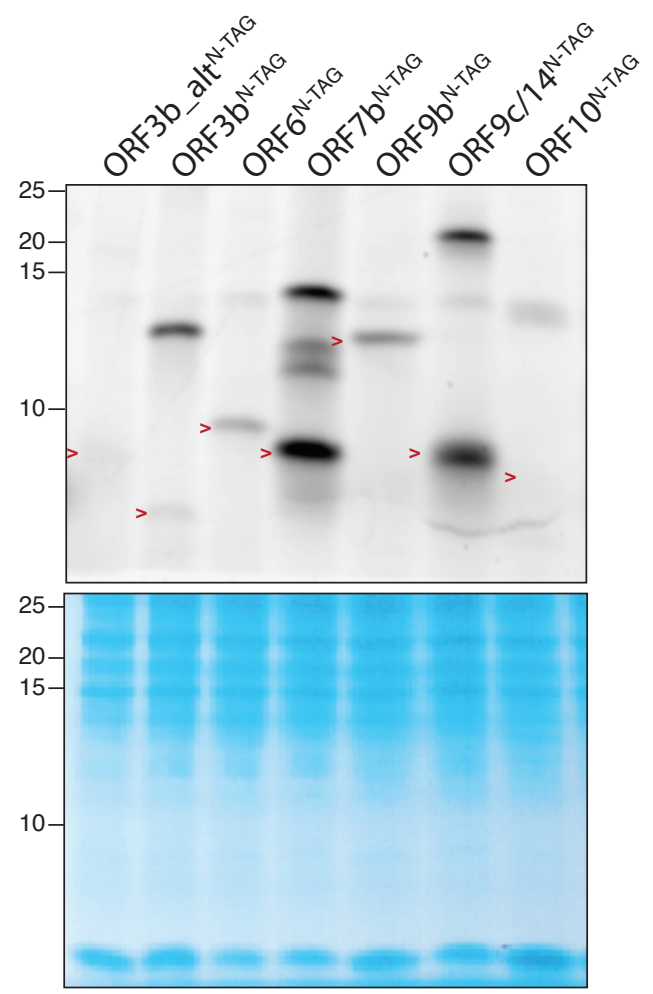


Supplementary Figure 12 - Labeling of SARS-CoV-2 derived microproteins in fixed cells. (A) COS-7 $7^{\text {PyIRS-AF }}$ cells were transfected with the indicated constructs and incubated for 24 hours with $50 \mu \mathrm{M}$ TCO*K. Cells were subsequently fixed, permeabilized and labeled with met-tet-BDP-FL and analyzed by confocal microscopy. SARS-CoV-2 M (Membrane) protein was used as a control because of its known localization to the Golgi. ${ }^{10}$ ORF7b is a type I (luminal N-terminus) transmembrane protein implicated as virulence factor, generated by leaky ribosome scanning in an alternative frame within the main ORF7a. ${ }^{11}$ The homologous microprotein in SARS1 is suggested to localize to the Golgi. ${ }^{12}$ Our observation is a wider distribution in Golgi, ER and plasma membrane. ORF9b is an alternative ORF within ORF9a and the SARS1 homolog is reported to localize to mitochondria. ${ }^{13}$ We also observe a distinct speckled pattern in the cytosol compatible with partial mitochondrial localization. ORF9c expression and function is currently unknown but we observe a distinct localization with cellular membranes. ORF3b of SARS-CoV-2 is truncated to a 22-amino acid fragment due to a premature stop codon as compared to the SARS1 homolog, but has been reported to potently suppress host cell antiviral interferon response. ${ }^{14}$ The truncation preserves a single predicted transmembrane domain and our imaging suggests an association with cellular membranes. (B) Tris-Tricine SDS-PAGE of additional SARS-CoV-2 STELLA N-terminal tagged microproteins and those used in A. ORF3b_alt (lane 1) is an alternative ORF proposed to exist within the main ORF3a, upstream of the ORF3b conserved amongst SARS viruses. ${ }^{15}$ However, we did not observe a stable labeled peptide. ORF10 is a hypothetical ORF whose translation has not been confirmed, and we also do not observe a product of the correct size. Several of the ORFs appear to yield higher molecular isoforms, some of which have also been observed when expressed with C-terminal 2xStrep-tag. ${ }^{15}$ 
Supplementary Figure 13 - movie stills

A

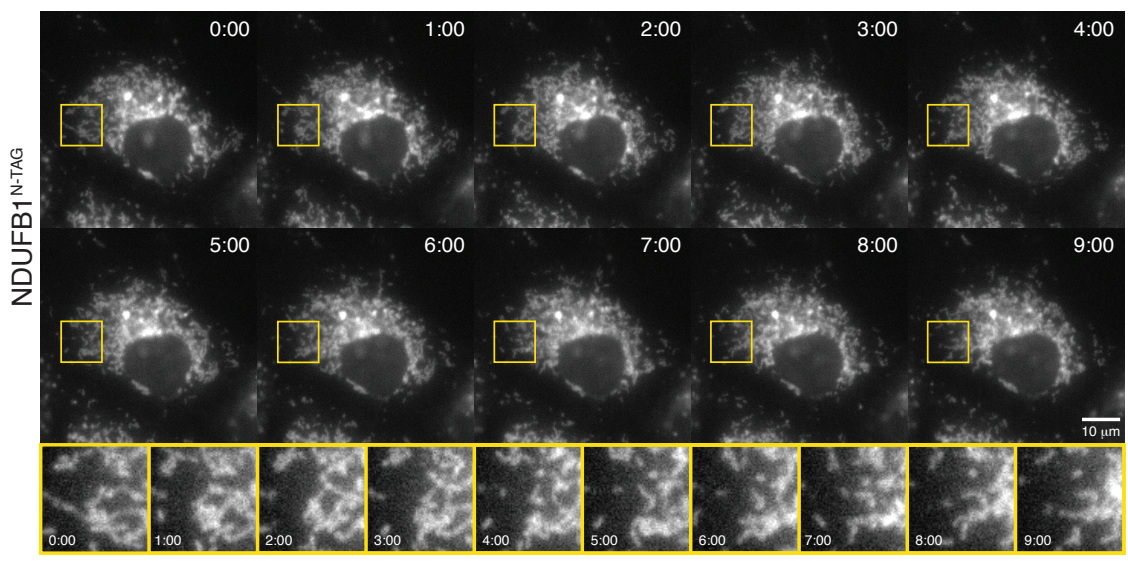

B

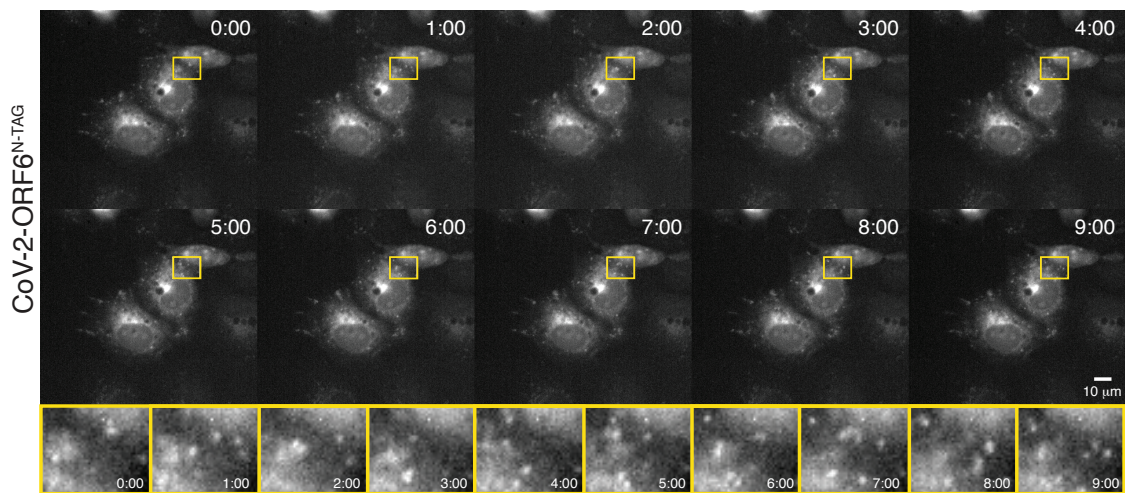


Supplementary Figure 13 - Still pictures of Supplementary Movie 1 and 2.

(A) Widefield, live cell imaging of NDUFB1 $1^{\mathrm{N}-\mathrm{TCO}^{*} \mathrm{~K}} .24$ hours after transfection and $\mathrm{TCO} * \mathrm{~K}$ incubation, COS-7 $7^{\text {PyIRS-AF }}$ cells were exposed for $30 \mathrm{~min}$ to $500 \mathrm{nM}$ me-tet-BDP-FL. Right after removal of the dye, cells were imaged over a short period of time to verify that the labeling approach was not toxic for the cells. In the movie, it is possible to appreciate the movement of NDUFB1-containing mitochondria. Timestamps (min:sec) are displayed in the top-right corner of each frame. (B) Widefield, live cell imaging of SARS-CoV-2-ORF6 ${ }^{\mathrm{N}-\mathrm{TCO}^{*} \mathrm{~K}}$. 24 hours after transfection and $\mathrm{TCO} * \mathrm{~K}$ incubation, COS-7 $7^{\text {PyIRS-AF }}$ cells were exposed for $30 \mathrm{~min}$ to $500 \mathrm{nM}$ me-tet-BDP-FL. Right after removal of the dye, cells were imaged over a short period of time. In the movie, it is possible to see ORF6-containing vesicles travelling through the cytoplasm. Timestamps (min:sec) are displayed in the top-right corner of each frame. 
Supplementary movie 1 - Widefield, live cell imaging of NDUFB1 ${ }^{\mathrm{N}-\mathrm{TCO}{ }^{* \mathrm{~K}}} .24$ hours after transfection and $\mathrm{TCO} * \mathrm{~K}$ incubation, COS-7 $7^{\text {PylRS-AF }}$ cells were exposed for $30 \mathrm{~min}$ to $500 \mathrm{nM}$ me-tet-BDP-FL. Right after removal of the dye, cells were imaged over a short period of time to verify that the labeling approach was not toxic for the cells. In the movie, it is possible to appreciate the movement of NDUFB1-containing mitochondria. Timestamps (min:sec) are displayed in the top-right corner of each frame.

Supplementary movie 2 - Widefield, live cell imaging of SARS-CoV-2-ORF6 ${ }^{\mathrm{N}-\mathrm{TCO}{ }^{* \mathrm{~K}}} .24$ hours after transfection and $\mathrm{TCO}^{*} \mathrm{~K}$ incubation, COS-7 $7^{\text {PylRs-AF }}$ cells were exposed for 30 min to $500 \mathrm{nM}$ me-tet-BDP-FL. Right after removal of the dye, cells were imaged over a short period of time. In the movie, it is possible to see ORF6-containing vesicles travelling through the cytoplasm. Timestamps (min:sec) are displayed in the top-right corner of each frame. 


\section{SUPPLEMENTARY REFERENCES}

(1) Meineke, B.; Heimgärtner, J.; Lafranchi, L.; Elsässer, S. J. Methanomethylophilus Alvus Mx1201 Provides Basis for Mutual Orthogonal Pyrrolysyl TRNA/Aminoacyl-TRNA Synthetase Pairs in Mammalian Cells. ACS Chem. Biol. 2018, 13, 3087-3096.

(2) Schindelin, J.; Arganda-Carreras, I.; Frise, E.; Kaynig, V.; Longair, M.; Pietzsch, T.; Preibisch, S.; Rueden, C.; Saalfeld, S.; Schmid, B.; et al. Fiji: An Open-Source Platform for Biological-Image Analysis. Nat. Methods 2012, 9, 676-682.

(3) McQuin, C.; Goodman, A.; Chernyshev, V.; Kamentsky, L.; Cimini, B. A.; Karhohs, K. W.; Doan, M.; Ding, L.; Rafelski, S. M.; Thirstrup, D.; et al. CellProfiler 3.0: Next-Generation Image Processing for Biology. PLoS Biol. 2018, 16, e2005970.

(4) Moggridge, S.; Sorensen, P. H.; Morin, G. B.; Hughes, C. S. Extending the Compatibility of the SP3 Paramagnetic Bead Processing Approach for Proteomics. J. Proteome Res. 2018, 17, 1730-1740.

(5) Elsässer, S. J. Generation of Stable Amber Suppression Cell Lines. Methods Mol. Biol. 2018, 1728, 237-245.

(6) Banaszynski, L. A.; Chen, L.-C.; Maynard-Smith, L. A.; Ooi, A. G. L.; Wandless, T. J. A Rapid, Reversible, and Tunable Method to Regulate Protein Function in Living Cells Using Synthetic Small Molecules. Cell 2006, 126, 995-1004.

(7) Li, C. H.; Tam, P. K. S. An Iterative Algorithm for Minimum Cross Entropy Thresholding. Pattern Recognit. Lett. 1998, 19, 771-776.

(8) Manders, E. M. M.; Verbeek, F. J.; Aten, J. A. Measurement of Co-Localization of Objects in Dual-Colour Confocal Images. J. Microsc. 1993, 169, 375-382.

(9) Costes, S. V.; Daelemans, D.; Cho, E. H.; Dobbin, Z.; Pavlakis, G.; Lockett, S. Automatic and Quantitative Measurement of Protein-Protein Colocalization in Live Cells. Biophys. J. 2004, 86, 3993-4003.

(10) Klumperman, J.; Locker, J. K.; Meijer, A.; Horzinek, M. C.; Geuze, H. J.; Rottier, P. J. Coronavirus M Proteins Accumulate in the Golgi Complex beyond the Site of Virion Budding. J. Virol. 1994, 68, 6523-6534.

(11) Pfefferle, S.; Krähling, V.; Ditt, V.; Grywna, K.; Mühlberger, E.; Drosten, C. Reverse Genetic Characterization of the Natural Genomic Deletion in SARS-Coronavirus Strain Frankfurt-1 Open Reading Frame $7 \mathrm{~b}$ Reveals an Attenuating Function of the $7 \mathrm{~b}$ Protein in-Vitro and in-Vivo. Virol. J. 2009, 6, 131.

(12) Schaecher, S. R.; Diamond, M. S.; Pekosz, A. The Transmembrane Domain of the Severe Acute Respiratory Syndrome Coronavirus ORF7b Protein Is Necessary and Sufficient for Its Retention in the Golgi Complex. J. Virol. 2008, 82, 9477-9491.

(13) Shi, C.-S.; Qi, H.-Y.; Boularan, C.; Huang, N.-N.; Abu-Asab, M.; Shelhamer, J. H.; Kehrl, J. H. SARS-Coronavirus Open Reading Frame-9b Suppresses Innate Immunity by Targeting Mitochondria and the MAVS/TRAF3/TRAF6 Signalosome. J. Immunol. 2014, 193, 3080-3089.

(14) Konno, Y.; Kimura, I.; Uriu, K.; Fukushi, M.; Irie, T.; Koyanagi, Y.; Sauter, D.; Gifford, R. J.; USFQ-COVID19 Consortium; Nakagawa, S.; Sato, K. SARS-CoV-2 ORF3b Is a Potent 
Interferon Antagonist Whose Activity Is Increased by a Naturally Occurring Elongation Variant. Cell Rep. 2020, 32, 108185.

(15) Gordon, D. E.; Jang, G. M.; Bouhaddou, M.; Xu, J.; Obernier, K.; White, K. M.; O’Meara, M. J.; Rezelj, V. V.; Guo, J. Z.; Swaney, D. L.; et al. A SARS-CoV-2 Protein Interaction Map Reveals Targets for Drug Repurposing. Nature 2020, 583, 459-468. 
UNCROPPED SOURCE IMAGE 
Figure 1
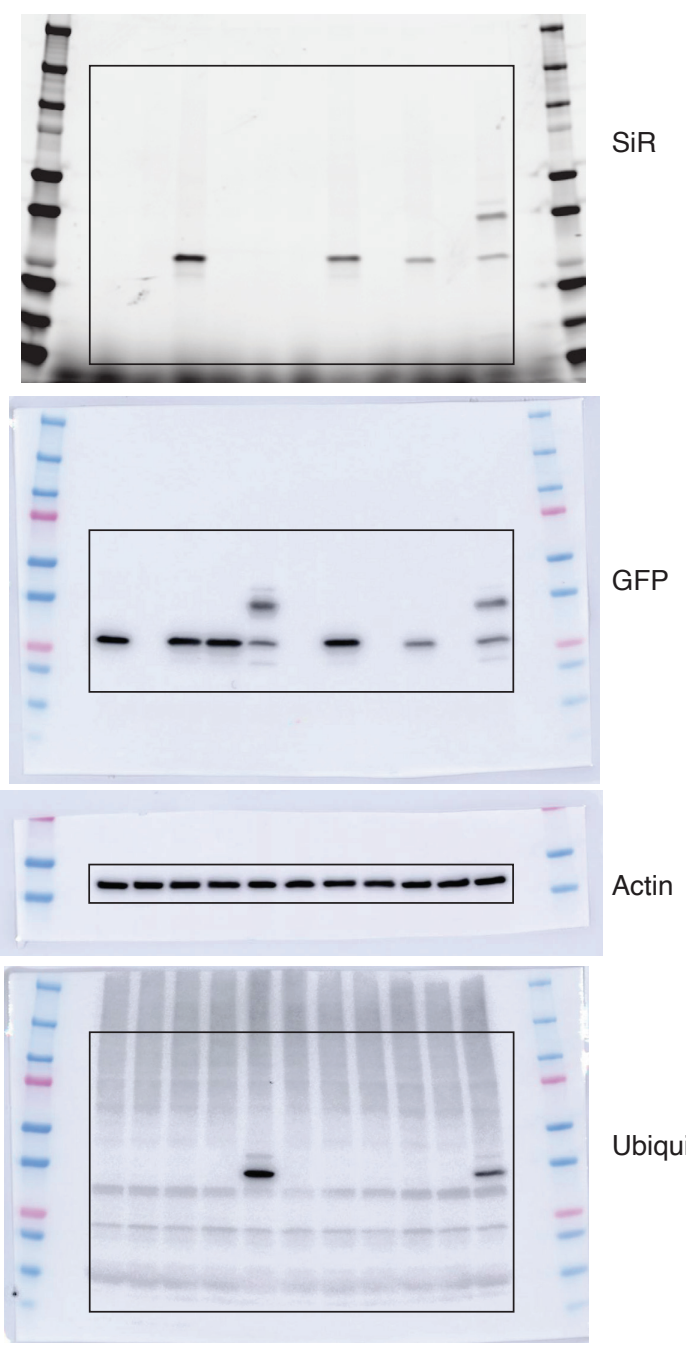

Ubiquitin

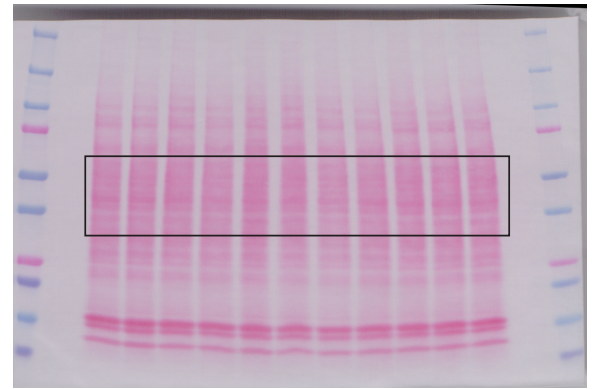

Ponceau 
Figure 2

A
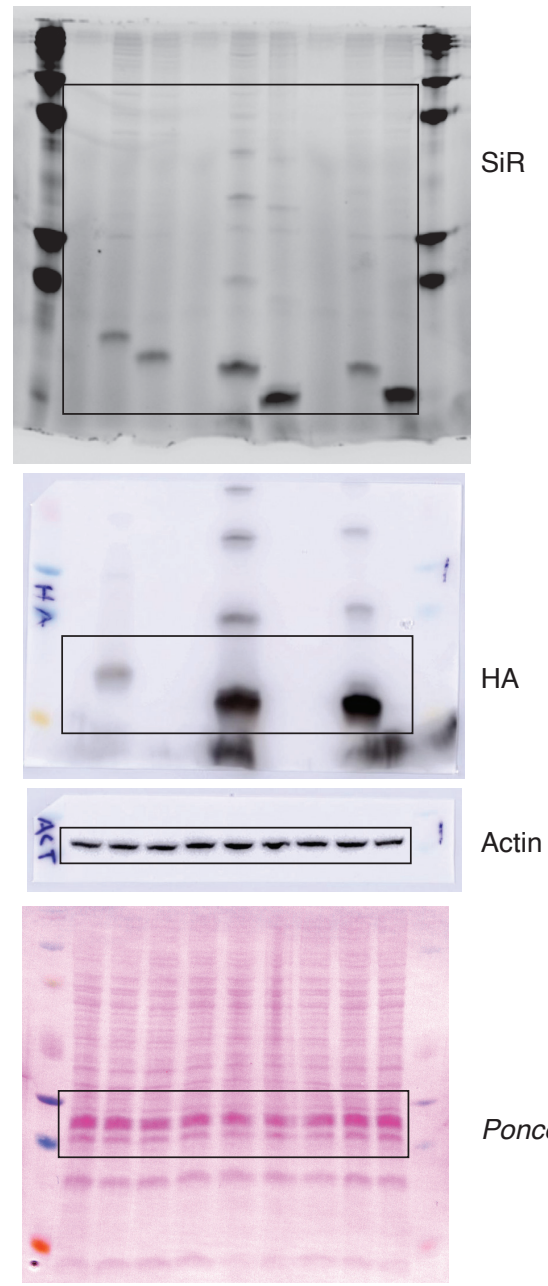

E
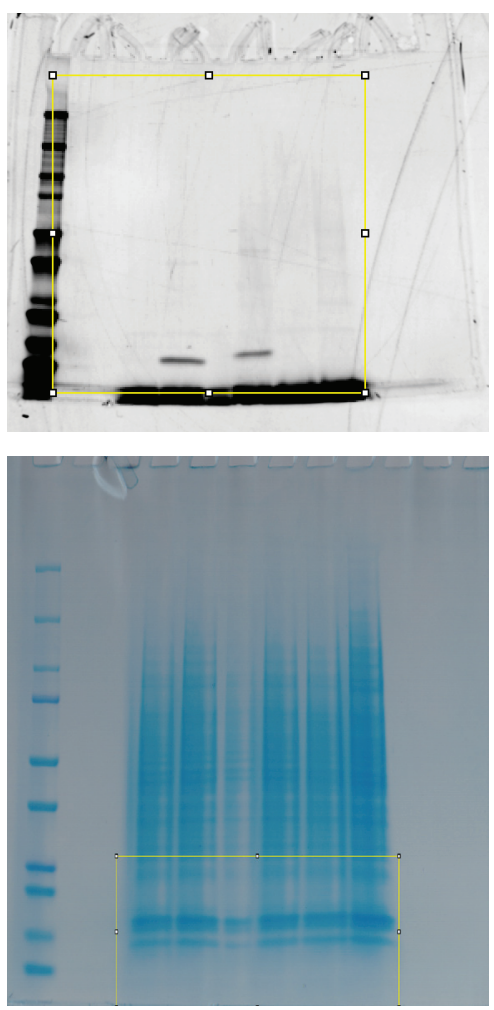

$\mathrm{SiR}$

D

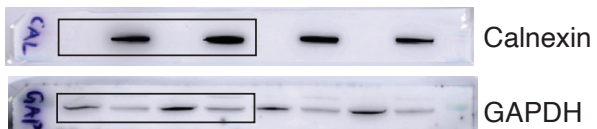

$\geqq \square-\square-\square A P D H$
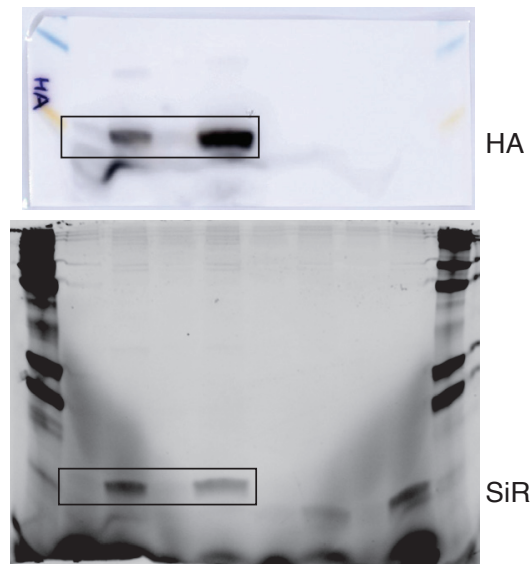

Ponceau

Coomassie 
Figure 3B

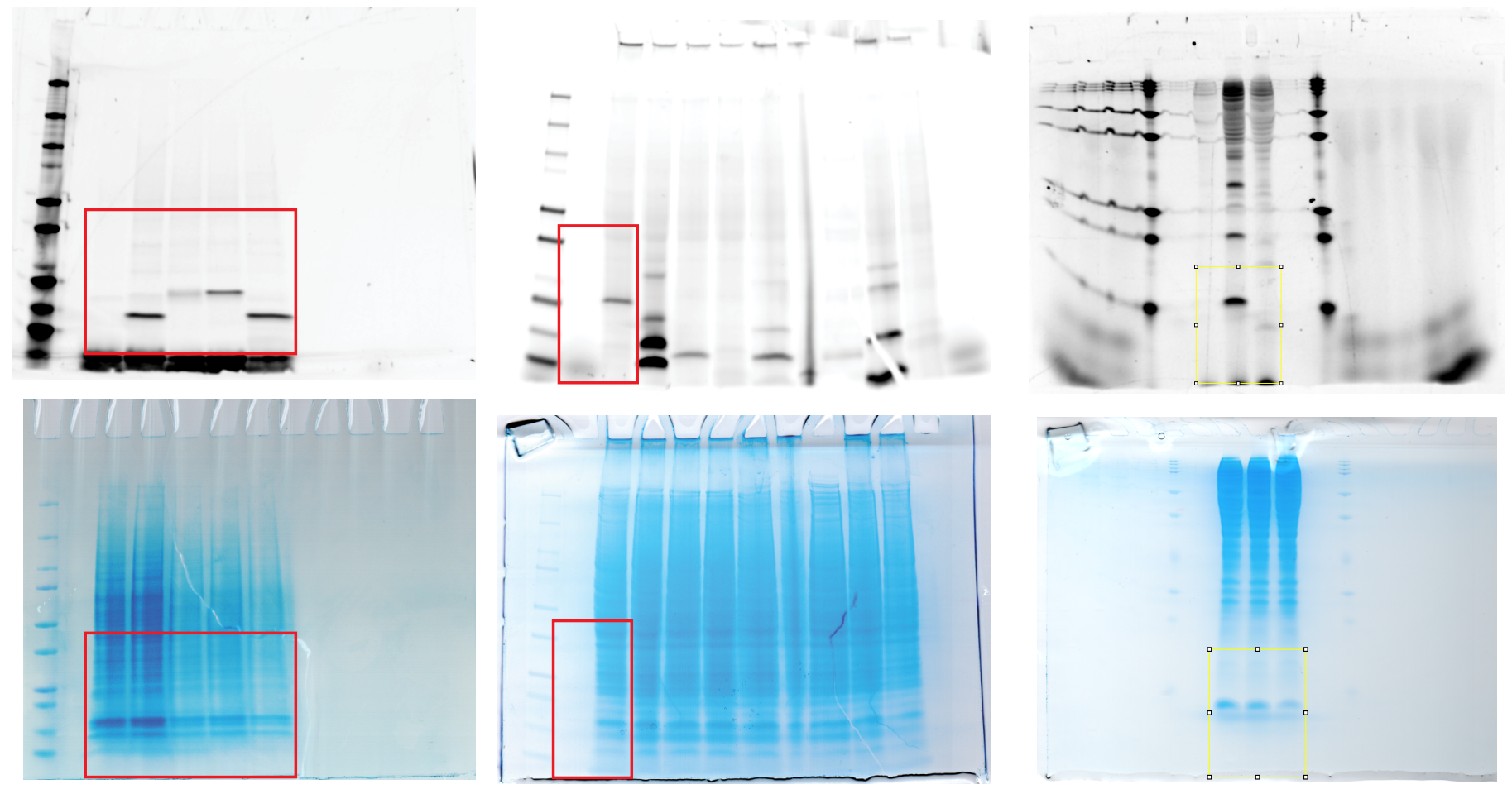


Supplementary Figure 1
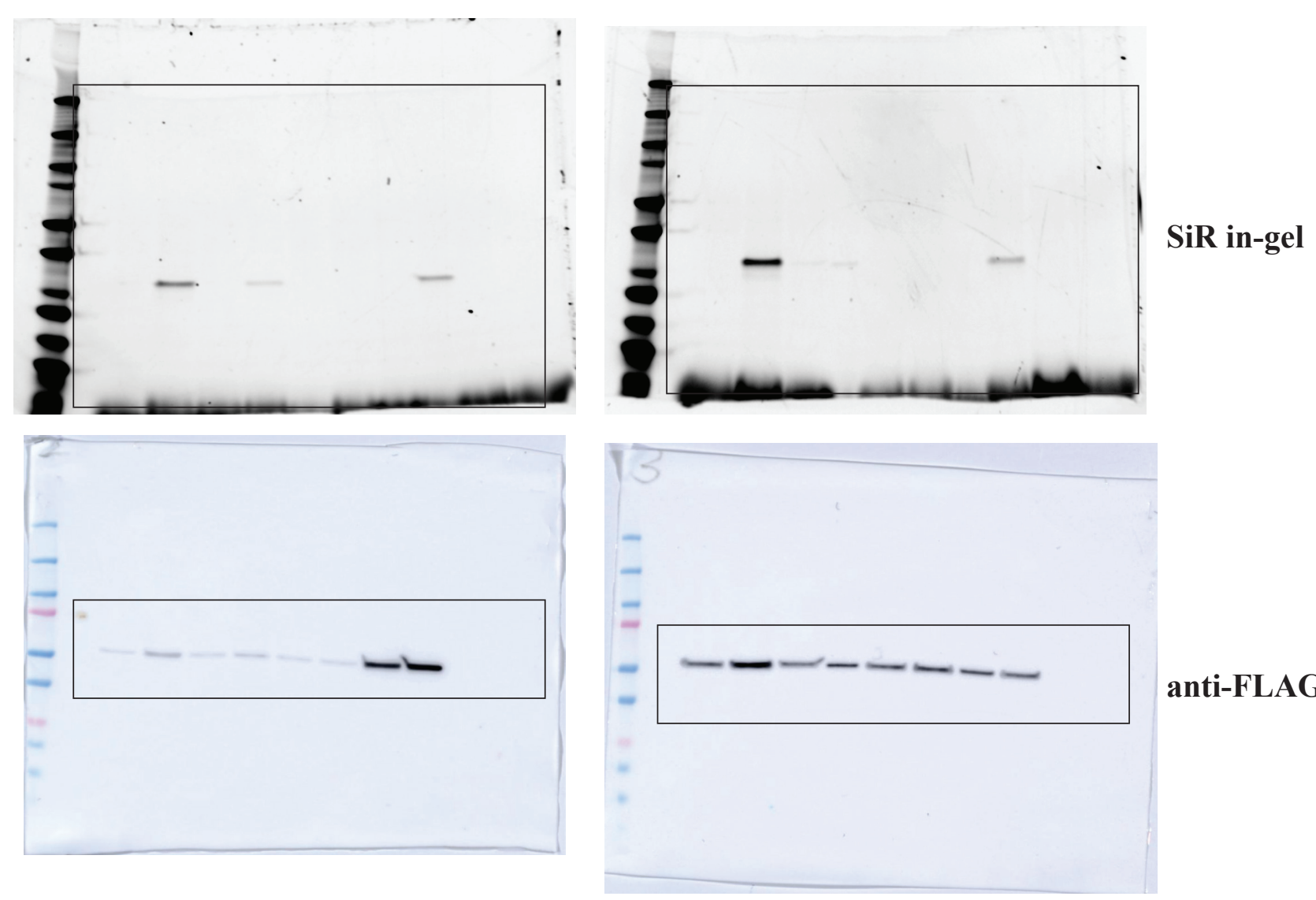

anti-FLAG
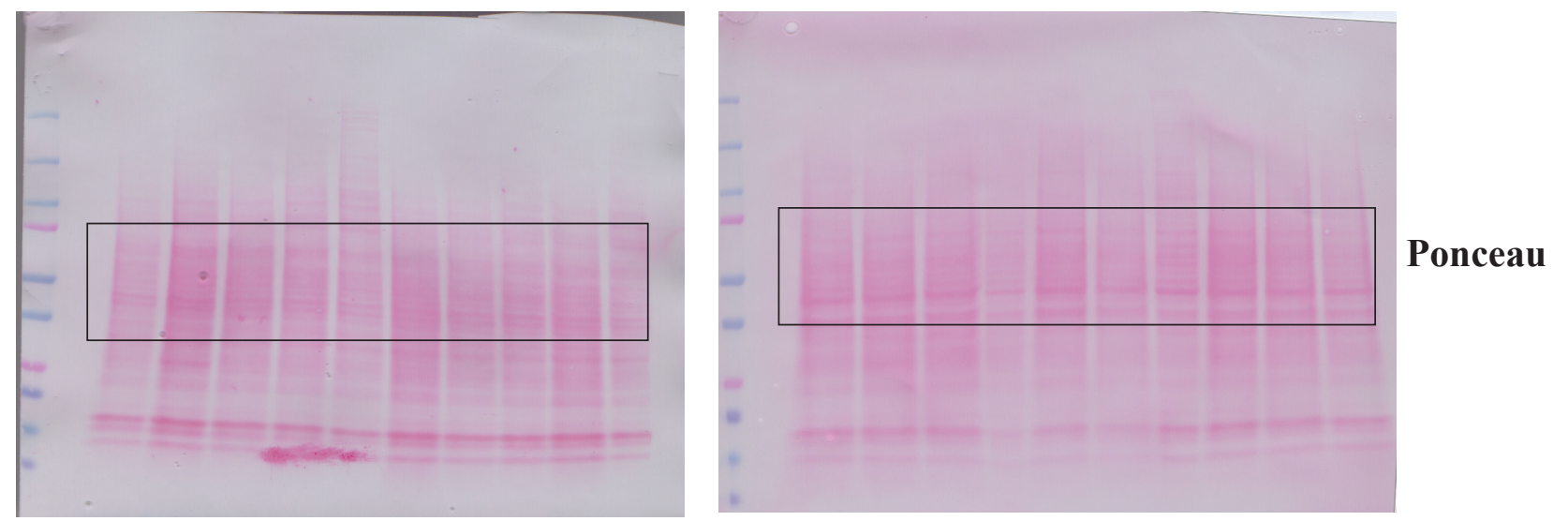
Supplementary Figure 5

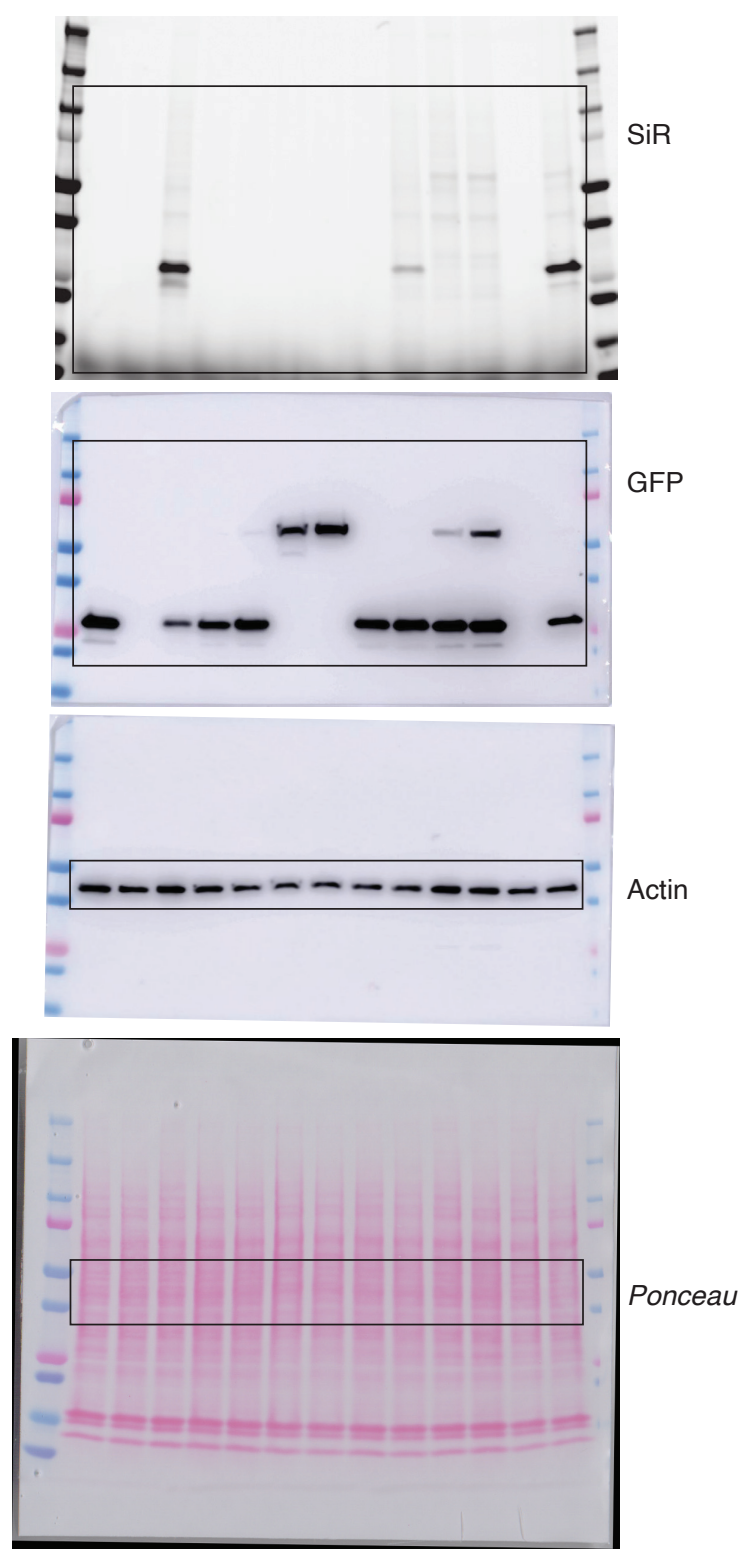


Supplementary Figure 6
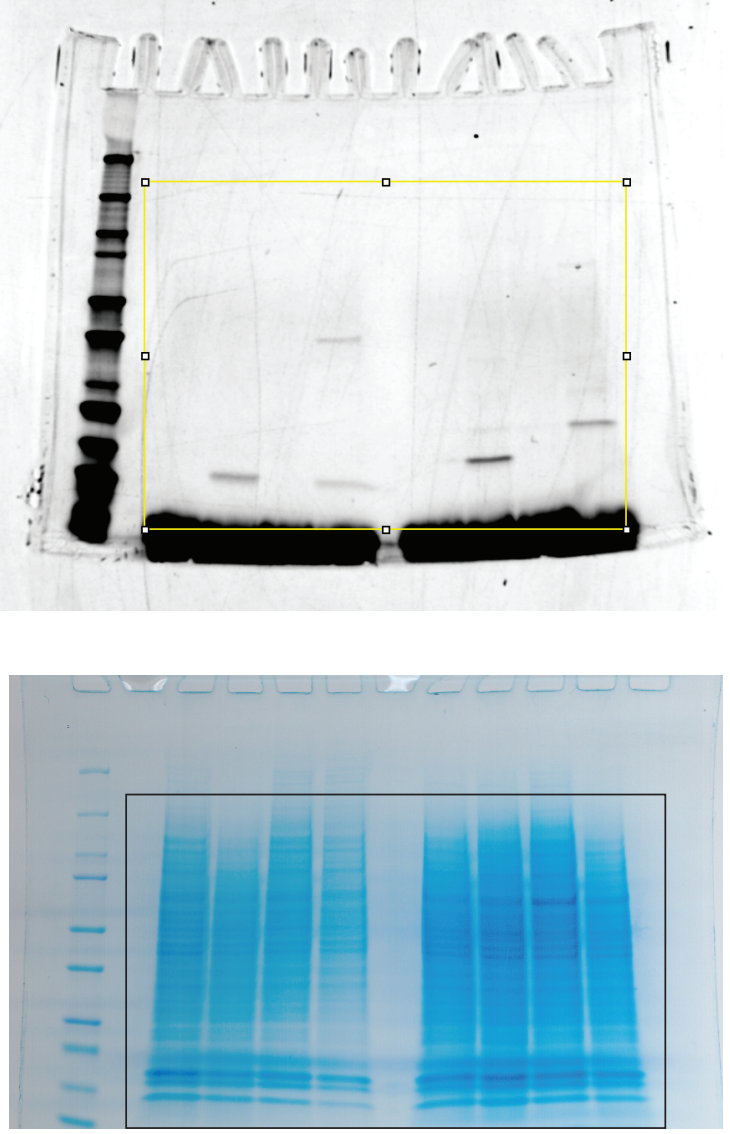
Supplementary Figure 7A

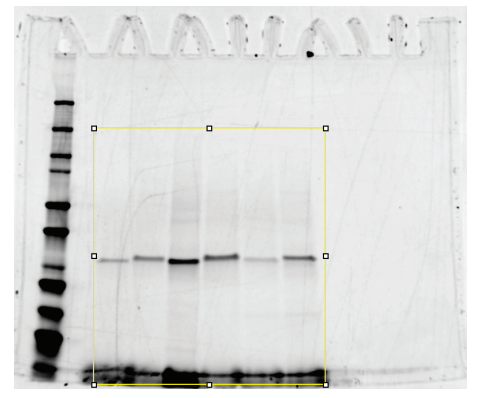

SiR

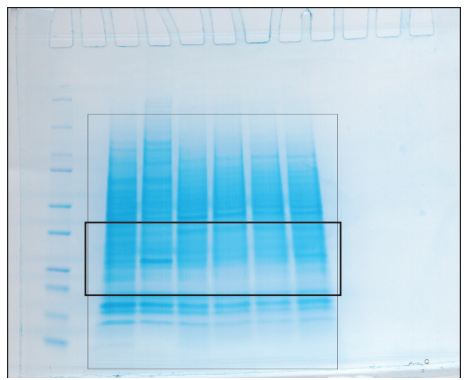

Supplementary Figure 7B
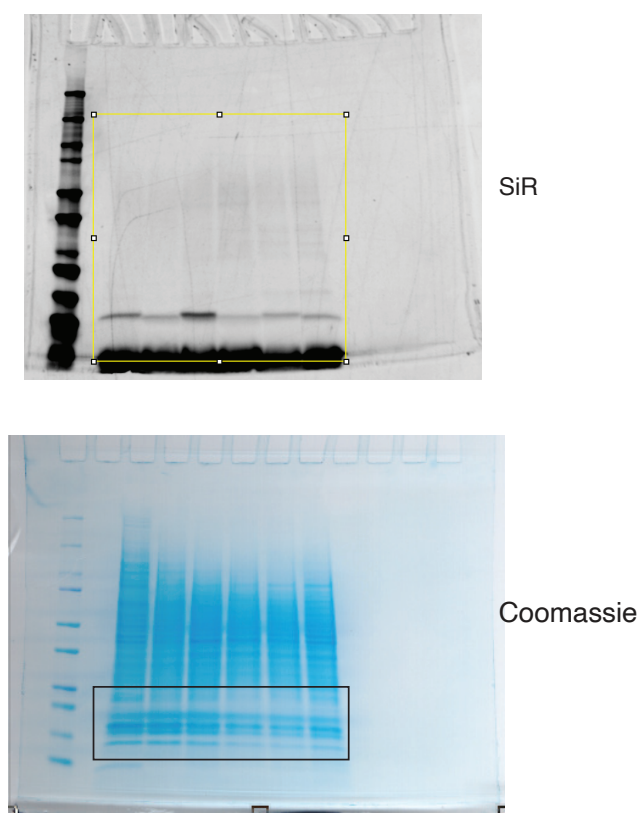
Supplementary Figure 11A
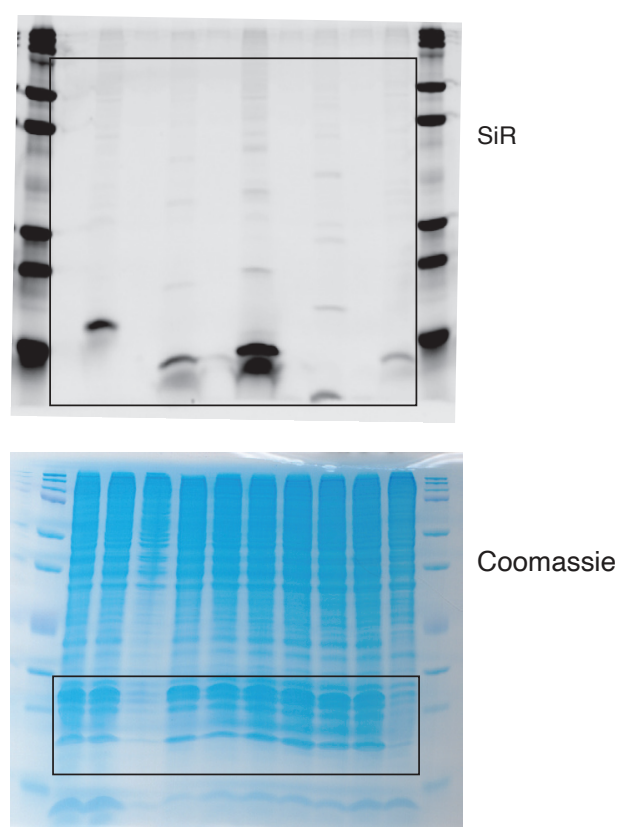

Supplementary Figure 11B
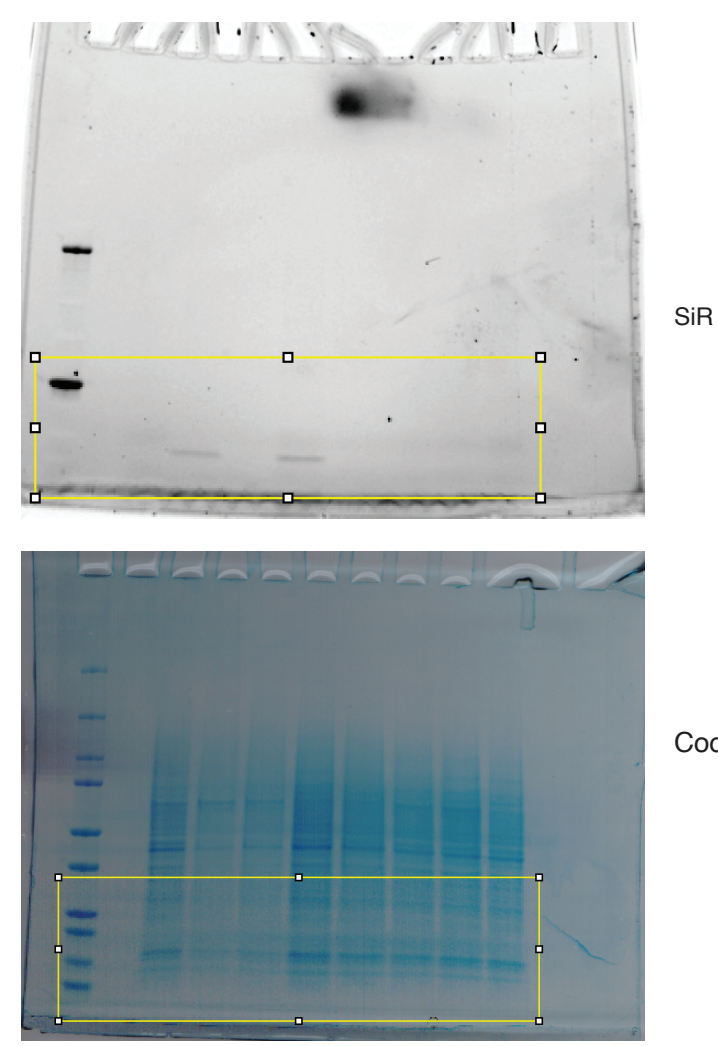

Coomassie 
Supplementary Figure 12B

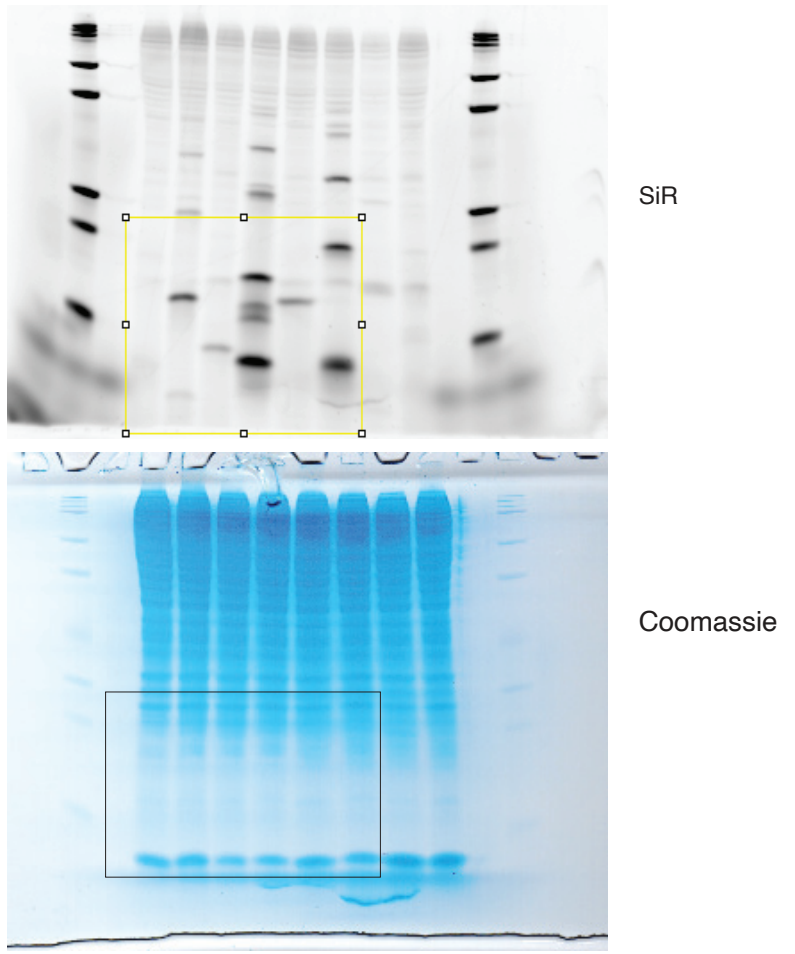

